\title{
Strong deflection limit of black hole gravitational lensing with arbitrary source distances
}

\author{
V. Bozza ${ }^{a, b}$, G. Scarpetta ${ }^{a, b, c}$ \\ a Dipartimento di Fisica "E.R. Caianiello", Università di Salerno, via Allende, I-84081 Baronissi (SA), Italy. \\ ${ }^{b}$ Istituto Nazionale di Fisica Nucleare, Sezione di Napoli. \\ ${ }^{c}$ International Institute for Advanced Scientific Studies, Vietri sul Mare (SA), Italy
}

(Dated: November 8, 2018)

\begin{abstract}
The gravitational field of supermassive black holes is able to strongly bend light rays emitted by nearby sources. When the deflection angle exceeds $\pi$, gravitational lensing can be analytically approximated by the so-called strong deflection limit. In this paper we remove the conventional assumption of sources very far from the black hole, considering the distance of the source as an additional parameter in the lensing problem to be treated exactly. We find expressions for critical curves, caustics and all lensing observables valid for any position of the source up to the horizon. After analyzing the spherically symmetric case we focus on the Kerr black hole, for which we present an analytical 3-dimensional description of the higher order caustic tubes.
\end{abstract}

PACS numbers: 95.30.Sf, 04.70.Bw, 98.62.Sb

Keywords: Relativity and gravitation; Classical black holes; Gravitational lensing

\section{INTRODUCTION}

Black holes have always attracted the interest of all physicists who hope to see General Relativity at work in a completely non-perturbative regime, outside any PostNewtonian expansion. Since most of the information we receive from black holes and their surroundings is in the form of electromagnetic waves, one of the fundamental problems to be faced is the propagation of such waves in a black hole spacetime. The situation can be possibly complicated by an accretion disk formed by neutral plasma 1]. For wavelengths at which observations are typically lead, the geometrical optics approximation provides a very robust description for the propagation of the light rays, defined as the lines orthogonal to the wavefronts. In all situations in which the plasma physics has little effect on the rays trajectories, the light rays simply follow null geodesics. Then all questions that involve the propagation of an electromagnetic signal require integration of the null geodesics equation. In the case of Kerr metric, the null geodesics have been expressed by Carter in terms of first integrals through the separation of the Hamilton-Jacobi equation [2]. Then, the integrals involved in these geodesics can be solved in terms of elliptic functions (see e.g. 3, 4]). Although these analytical solutions are not particularly illuminating by themselves, they can be successfully employed to build fast and accurate numerical codes $[3,4,4,5,6]$, by which one can get particular answers, such as the shape of the iron K-line or the appearance of the accretion flow into the black hole in a future hypothetical high-resolution image [7, 8, 9].

The problem of finding the null geodesics connecting a source to an observer in a curved background is usually referred to as gravitational lensing. It has been pointed out by many authors that in a black hole spacetime there are infinitely many possible trajectories for the photons emitted by a point-source to reach the observer 17, 10, 12, 13, 14, 15, 16]. For each of these trajecto- ries the observer will detect an independent image of the original source. The images can be classified according to the number of loops performed by the corresponding photons around the black hole. One starts from the primary and secondary image, which are formed by photons performing no loops. These are already present in the classical weak deflection limit of gravitational lensing. Besides these, there are two infinite sequences of higher order images, formed by photons performing one or more loops around the black hole before reaching the observer. These images are progressively fainter and appear closer and closer to the apparent shadow cast by the black hole on the sky.

The higher order images contribute much less than the primary and secondary images to the total flux and are often completely masked in situations in which they are not separable from the main images [17]. Therefore, the best chances to observe higher order images are offered by a black hole as massive and close to us as possible, so that its apparent angular size is the largest. The natural candidate is the black hole at the center of our Galaxy, identified with the radio-source $\mathrm{Sgr} \mathrm{A}^{*}$. This is believed to be a $3.6 \times 10^{6} M_{\odot}$ supermassive black hole slowly accreting material from the surrounding environment [18, 19]. Its distance is $D_{O L}=8 \mathrm{kpc}$, so that the Schwarzschild radius $R_{S c h}$ of this black hole spans an angle of roughly $9 \mu$ as in our sky. A resolution of this order of magnitude is needed to detect the higher order images, besides the requirement of negligible absorption in the wavelength of the emitted signal by the surrounding material. In spite of these difficulties, the detection of higher order images of sources around $\mathrm{Sgr} \mathrm{A}^{*}$ should be at hand of future interferometers operating in the sub-mm range, where one expects to detect higher order images of bright spots on the accretion disk [9], or in the X-ray band, where Low Mass X-Ray Binaries and other sources are active 3, 20, 21, 22]. Such images would be invaluable witnesses of the strong gravitational field around the supermassive 
black hole at the center of our Galaxy; their direct observation would thus be of striking importance in the confirmation of our gravitational theory.

In addition to the considerations about their importance, higher order images can boast two advantages with respect to lower order ones: if the black hole has non-negligible spin, they can easily form large arcs and additional images because their caustics have a larger angular extension compared to the first caustic; secondly, they enjoy a relatively much simpler analytical description.

The treatment of higher order images can take advantage of the fact that the deflection diverges logarithmically when the impact parameter reaches a minimum value. Then, the higher order images can be obtained by a simplified lens equation where the deflection terms are replaced by the first terms of their expansions in terms of the impact parameter. This procedure is analogous to the weak deflection limit but sets its starting point in the opposite regime and is thus conventionally called strong deflection limit. It was firstly used by Darwin in 1959 for the Schwarzschild black hole [10] and then revived several times [7, 12, 13, 14] until it was generalized to all spherically symmetric black holes [23]. This method was then applied to several interesting black hole metrics, also motivated by string and alternative theories [24, 25, 26]. The time delay calculation for higher order images was done in Ref. [27]. The method was recently extended to the presence of external shear fields in a setup analogous to the Chang \& Refsdal lens [28]. The extension to Kerr metric has required several steps, starting from the purely equatorial case [29] to the case of generic trajectories with equatorial observers [21], and finally to the general case [22] (in the latter two works, the treatment is however limited to the second order in the black hole spin). An application to the Kerr-Sen dilaton-axion black hole has also been performed [30]. Recently Iyer and Petters have found an alternative expansion parameter that significantly reduces the discrepancy between the strong deflection limit and the exact deflection formula [31]. They have also explored the next to leading order terms in the strong deflection expansion.

The strong deflection limit allows a simple analytic investigation of the gravitational lensing properties of any black hole metric in a well-defined limit, in which the results are easily comparable from one metric to another. For the Kerr metric it has been able to provide the first analytical formulae for the caustics and the critical curves involving higher order images and for lensing of sources near caustics. However, in the formulation used up to now, it has just been applied to sources very far from the black hole, so that the limit $D_{L S} \gg R_{S c h}$ has been taken in all equations (here $D_{L S}$ is the distance of the source from the black hole and $R_{S c h}=2 G M / c^{2}$ is the Schwarzschild radius of the black hole).

The purpose of this paper is to remove the limitation to very far sources, enlarging the investigation of gravitational lensing in the strong deflection limit to sources placed at arbitrary distances from the black hole. We will thus be able to discuss the mathematical structure of the lensing problem and all the lensing observables taking $D_{L S}$ as an additional parameter. We will show that the strong deflection limit is well-defined even for sources inside the photon sphere, so that our discussion can be safely pushed up to sources lying just outside the horizon of the black hole. Similarly, in the Kerr metric we will be able to describe the caustic hypersurface from infinite radial distance up to the horizon.

This paper is structured as follows. Sect. II contains the new outline of the strong deflection limit for spherically symmetric black holes with arbitrary source position; it analyzes the lens equation and observables and discusses the Schwarzschild metric as a simple example. Sect. III contains the extension of Kerr black hole lensing to arbitrary source distances, with some details moved to the appendix. Section [V] contains the conclusions.

\section{SPHERICALLY SYMMETRIC BLACK HOLES}

In this section we shall present an updated version of the method outlined in Ref. [23]. Besides including the finiteness of source and observer distances from the black hole, we also make some more slight refinements that allow further generalization of the method and more physical insight. We stress the importance of the study of spherically symmetric black holes as propaedeutic to the investigation of the Kerr metric, to be tackled in the next section.

As in Ref. 23], we start from three basic assumptions on the spacetime metric:

a) The spacetime is stationary and spherically symmetric, so that the line element can be written in the form

$$
d s^{2}=A(r) d t^{2}-B(r) d r^{2}-C(r)\left(d \vartheta^{2}+\sin ^{2} \vartheta d \phi^{2}\right) .
$$

b) We assume that our metric is asymptotically flat, so that for $r \rightarrow \infty$ we have

$$
A(r) \rightarrow 1, \quad B(r) \rightarrow 1, \quad C(r) \rightarrow r^{2} .
$$

c) Furthermore, we assume that the function $C(r) / A(r)$ has one minimum at $r_{m}>0$, corresponding to the radius of the photon sphere $r_{m}$ [32].

In some gravitational theories, the photons do not follow geodesics of the background geometry, but the selfinteraction makes them follow geodesics with respect to some effective metric [25]. These particular cases can fit into our treatment, provided that one uses the effective metric felt by the photons. Assumption (b) can be generalized to spacetimes that are asymptotically conformal to flat, thanks to the conformal invariance of null geodesics. In this way one can include e.g. black holes with a cosmological constant [26].

Let us give a closer look at assumption (c) and specifically at the function $C / A$. We can note that asymptotic 


\section{$\mathrm{C} / \mathrm{A}$}

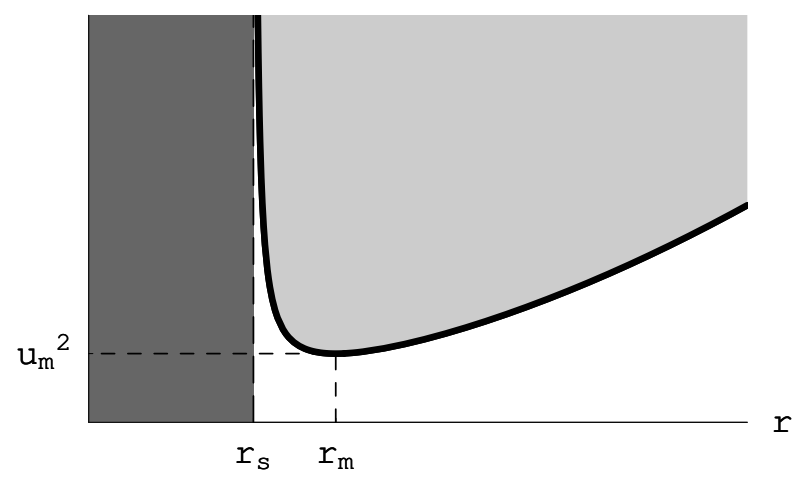

FIG. 1: The function $C(r) / A(r)$ plotted for the Schwarzschild metric $\left(A=1-R_{S c h} / r, C=r^{2}\right)$. In this case the static limit is $r_{s}=R_{S c h}$ and the photon sphere radius is $r_{m}=1.5 R_{S c h}$. The light-gray region is forbidden because it corresponds to imaginary $\dot{r}$. The dark-gray region lies below the horizon of the black hole and is uninteresting for gravitational lensing.

flatness drives the function $C / A$ to approach $r^{2}$ at very large $r$. Moreover, in any metric admitting a static limit $r_{s}$, such that $A\left(r_{s}\right)=0$, the function $C / A$ diverges at $r_{s}$. So, by continuity it must have at least one minimum greater than $r_{s}$. As a useful reference, in Fig. 1, we plot the function $C / A$ for the Schwarschild spacetime, where it assumes the form $r^{3} /\left(r-R_{S c h}\right)$. Following Ref. [32], all stationary points of $C / A$ are technically photon spheres. However, maxima are not significant for gravitational lensing, since they are only accessible to locally emitted photons. We are actually interested in minima of $C / A$, since they give rise to logarithmic divergences in the deflection angle, as it will be clear later. Although it is relatively easy to find metrics which also develop a maximum in $C / A$ (e.g. Reissner-Nordström with superextremal charge), it seems difficult to imagine a metric developing a second minimum. Such a metric would require a quite exotic source to be sustained as a viable solution of gravitational equations. Through assumption (c), we are discarding these too problematic spacetimes and stick to more reasonable metrics with only one minimum for $C / A$.

Now let us start the calculation of the photon deflection. The spherical symmetry allows us to choose the equatorial plane as the plane where the entire motion of the photon takes place, so that $\vartheta=\pi / 2$ and $\dot{\vartheta}=0$, where the dot denotes derivative with respect to the affine parameter.

The dynamics of the photon can be derived from the Lagrangian (see e.g. 33] for a complete discussion of null geodesics in Schwarzschild and Kerr spacetimes)

$$
\mathcal{L}=-\frac{1}{2} g_{\mu \nu} \dot{x}^{\mu} \dot{x}^{\nu}
$$

The coordinates $t$ and $\phi$ are cyclic so that their conjugate momenta are constants of motion. They can be identified with the specific energy $E$ and angular momentum $J$

$$
\begin{aligned}
& E=A(r) \dot{t} \\
& J=C(r) \dot{\phi} .
\end{aligned}
$$

We can choose the orientation of the polar axis so that $J>0$.

The last constant of motion comes from the fact that the photon moves along null geodesics of the metric (1) so that $g_{\mu \nu} \dot{x}^{\mu} \dot{x}^{\nu}=0$. From this equation, we derive the expression of $\dot{r}$ :

$$
\dot{r}= \pm \frac{E}{\sqrt{B C}} \sqrt{\frac{C}{A}-\frac{J^{2}}{E^{2}}} .
$$

The angle formed by the spatial components of the photon momentum $p^{i}=(\dot{r}, \dot{\phi})$ with a normalized vector tangent to a sphere centered on the black hole $t^{i}=$ $(0,1 / \sqrt{C})$ is

$$
\vartheta=\arccos \frac{-g_{i j} t^{i} p^{j}}{|p||t|}=\arccos \left(\frac{J}{E} \sqrt{\frac{A}{C}}\right) .
$$

So, in any point of the photon trajectory, the knowledge of the value of the combination

$$
u=\frac{J}{E},
$$

allows us to calculate the angles formed by the photon with respect to the radial and tangent directions. The photon moves radially if $u=0$ and tangentially in points such that $u=\sqrt{C / A}$. It is also easy to prove that for those photons reaching the asymptotic flat region, this quantity equals the impact parameter, defined as the distance between the black hole and the asymptotic trajectory followed by the photon. On the basis of its immediate connection with the observed direction of the photon, we will eliminate $J$ in favor of $u$, where possible.

Inversion of the radial motion can occur only at the points that make the argument of the square root vanish in Eq. (6), which correspond to points of instantaneous tangential motion by virtue of Eq. (7). However, assumption (c) states that the function $C / A$ has a single minimum at $r_{m}$. So, a quick look at Fig. 1 convinces that the equation

$$
\frac{C\left(r_{0}\right)}{A\left(r_{0}\right)}=u^{2},
$$

has real roots only if $u>u_{m}$, with

$$
u_{m}=\sqrt{\frac{C_{m}}{A_{m}}},
$$

where we have introduced the short notation $A_{m} \equiv$ $A\left(r_{m}\right)$ and similarly for $B$ and $C$.

At this point it is convenient to distinguish the case $D_{L S}>r_{m}$ (source outside the photon sphere) from the case $D_{L S}<r_{m}$ (source inside the photon sphere). We 
shall finally find that they both lead to the same expression for the deflection of a photon in the strong deflection limit, given by Eq. (51). The next two subsections deal with the details of the calculations in the two mentioned cases.

\section{A. Source outside the photon sphere}

Let us analyze all possibilities for the radial motion of a photon emitted by a source outside the photon sphere.

Some photons will leave the source with positive $\dot{r}$. Since the photons are emitted at $D_{L S}>r_{m}$, these photons never meet inversion points and run towards the asymptotic region without experiencing any effective deflection by the black hole. If $D_{O L}>D_{L S}$ some of them reach the observer and give rise to the primary image, which is not the subject of our analysis anyway.

Some other photons leave the source with negative $\dot{r}$. If $D_{O L}<D_{L S}$, there is still the possibility that some of them can reach the observer without inverting their motion and form the primary image. But some other photons do not meet the observer and run towards the black hole. The photons with $u<u_{m}$ will inexorably sink into the black hole, since Eq. (9) will admit no real roots. If $u>u_{m}$, the photons invert their motion at the largest root of Eq. (9), which we indicate by $r_{0}$ and identify with the closest approach distance. After the inversion in the radial motion, these photons go back towards the asymptotic region and eventually reach the observer, giving rise to the secondary and higher order images.

All these considerations can be summarized by saying that light rays shot at too small impact parameters are swallowed by the black hole, whereas those shot at larger impact parameters are just deflected, the limiting value of $u$ being $u_{m}$. Our objective is to quantify the deflection of these photons as a function of $u$.

The azimuthal shift of the photon is

$$
\Delta \phi=\int_{\phi_{i}}^{\phi_{f}} d \phi=\int_{D_{L S}}^{r_{0}} \frac{\dot{\phi}}{\dot{r}} d r+\int_{r_{0}}^{D_{O L}} \frac{\dot{\phi}}{\dot{r}} d r
$$

where we have separated the motion of the photon into approach phase (with $r$ running from $D_{L S}$ to the inversion point $r_{0}$ ) and departure phase (with $r$ running from $r_{0}$ to $\left.D_{O L}\right)$. In the first integral we use the expression for $\dot{r}$ with the minus sign, in the second integral we use the expression with the plus sign. Using Eqs. (5) and (6), and reversing the extrema in the first integral, we have

$$
\begin{aligned}
\Delta \phi & =\Delta \phi_{S}+\Delta \phi_{O} \\
\Delta \phi_{i} & =\int_{r_{0}}^{D_{L i}} u \sqrt{\frac{B}{C}}\left(\frac{C}{A}-u^{2}\right)^{-1 / 2} d r,
\end{aligned}
$$

with the short notation $i=O, S$ and $D_{L i}=D_{O L}, D_{L S}$.
Note that the integrand diverges at $r_{0}$, which is defined as the largest root of the last factor. In order to study the character of the divergence, it is opportune to perform a detailed analysis of the function

$$
R(r, u)=\frac{C(r)}{A(r)}-u^{2}
$$

which governs the divergence of the integrand in the lower extremum. From the previous discussion, we know that $R(r, u)$ has a minimum at $r_{m}$ for any fixed value of $u$; it vanishes at $\left(r_{m}, u_{m}\right)$ by the definition of $u_{m}$; it vanishes at $\left(r_{0}, u\right)$ by the definition of $r_{0}$. These properties can be formalized by the equations

$$
\begin{aligned}
& \frac{\partial R}{\partial r}\left(r_{m}, u_{m}\right)=0 \\
& R\left(r_{m}, u_{m}\right)=0 \\
& R\left(r_{0}, u\right)=0 .
\end{aligned}
$$

Since we are interested into those trajectories whose inversion point is very close to the minimum $r_{m}$, we define a parameter $\delta$ by the equation

$$
r_{0}=r_{m}(1+\delta)
$$

Correspondingly, also the impact parameter must be very close to the minimum. We thus define the parameter $\epsilon$ by the equation

$$
u=u_{m}(1+\epsilon)
$$

Then, inserting (18) and (19) into Eq. (17) and expanding to the lowest order in $\delta$ and $\epsilon$, we have

$$
\begin{aligned}
& 0=R\left(r_{m}, u_{m}\right)+\frac{\partial R}{\partial r}\left(r_{m}, u_{m}\right) r_{m} \delta \\
& +\frac{1}{2} \frac{\partial^{2} R}{\partial r^{2}}\left(r_{m}, u_{m}\right) r_{m}^{2} \delta^{2}+\frac{\partial R}{\partial u}\left(r_{m}, u_{m}\right) u_{m} \epsilon .
\end{aligned}
$$

The first two terms vanish because of Eqs. (15) and (16) and we are left with a simple relation between $\delta^{2}$ and $\epsilon$, which tells us how much the inversion point $r_{0}$ differs from the photon sphere radius $r_{m}$, when we increase the impact parameter of the photon from the minimum value $u_{m}$ to $u$. Given the form of $R(r, u)$ it simply reads

$$
\epsilon=\frac{\beta_{m}}{2 u_{m}^{2}} \delta^{2}
$$

with

$$
\beta_{m}=\frac{1}{2} \frac{\partial^{2} R}{\partial r^{2}}\left(r_{m}, u_{m}\right) r_{m}^{2}=\frac{1}{2} r_{m}^{2} \frac{C_{m}^{\prime \prime} A_{m}-A_{m}^{\prime \prime} C_{m}}{A_{m}^{2}},
$$

where the prime denotes derivative with respect to the argument and the subscript $m$ means that the result must be evaluated at $r_{m}$ as usual. Thus we obtain that $\epsilon$ is of the same order as $\delta^{2}$. 
Let us analyze the behavior of $R(r, u)$ when $r$ is very close to $r_{0}$. We introduce the parametrization

$$
r=\frac{r_{0}}{1-\eta}
$$

with $0 \leq \eta<1$, and study $R(r(\eta), u)$ for small values of $\eta$. Expanding to the second order in $\eta, \delta$, and first order in $\epsilon$, we find

$$
\begin{aligned}
& R(\eta, u) \simeq R\left(r_{m}, u_{m}\right)+r_{m} \frac{\partial R}{\partial r}\left(r_{m}, u_{m}\right)\left(\delta+\eta+\eta \delta+\eta^{2}\right) \\
& +\frac{1}{2} r_{m}^{2} \frac{\partial^{2} R}{\partial r^{2}}\left(r_{m}, u_{m}\right)(\delta+\eta)^{2}+\frac{\partial R}{\partial u}\left(r_{m}, u_{m}\right) u_{m} \epsilon .
\end{aligned}
$$

Again, the first two terms vanish because of Eqs. (15) and (16). Moreover, the $\epsilon$-term cancels with the remaining $\delta^{2}$-term because of Eq. (20). The behavior of $R(\eta, u)$ for small $\eta$ is thus

$$
R(\eta, u)=\beta_{m}\left(2 \delta \eta+\eta^{2}\right)+o\left(\eta^{2}\right)
$$

Now that we have found the dominant terms in $R(\eta, u)$ for $\eta$ close to 0 , which corresponds to $r$ close to $r_{0}$, we can return to the integral in Eq. (13). Changing the integration variable from $r$ to $\eta$, the integration ranges become $\left[0, \eta_{i}\right]$, where $\eta_{i}=1-r_{0} / D_{L i}$. Each integral assumes the form

$$
\Delta \phi_{i}=\int_{0}^{\eta_{i}} u \sqrt{\frac{B(\eta)}{C(\eta)}}[R(\eta, u)]^{-1 / 2} \frac{r_{0}}{(1-\eta)^{2}} d \eta
$$

Now we add and subtract a term containing the divergence of the integrand at small values of $\eta$. We then separate the integral into two parts:

$$
\begin{aligned}
& \Delta \phi_{i}=I_{D}+I_{R} \\
& I_{D}=\int_{0}^{\eta_{i}} \frac{u_{m}}{\sqrt{\beta_{m}}} \sqrt{\frac{B_{m}}{C_{m}}} \frac{r_{m}}{\sqrt{2 \delta \eta+\eta^{2}}} d \eta \\
& I_{R}=\int_{0}^{\eta_{i}}\left[u \sqrt{\frac{B(\eta)}{C(\eta)}}[R(\eta, u)]^{-1 / 2} \frac{r_{0}}{(1-\eta)^{2}}\right. \\
& \left.-\frac{u_{m}}{\sqrt{\beta_{m}}} \sqrt{\frac{B_{m}}{C_{m}}} \frac{r_{m}}{\sqrt{2 \delta \eta+\eta^{2}}}\right] d \eta .
\end{aligned}
$$

Of course, the sum of the two integrals $I_{D}$ and $I_{R}$ is just the original integral (26), but now the first integral is elementary and reads

$$
I_{D}=r_{m} \sqrt{\frac{B_{m}}{A_{m} \beta_{m}}} \log \frac{\eta_{i}+\delta+\sqrt{\eta_{i}\left(\eta_{i}+2 \delta\right)}}{\delta}
$$

The divergence for $\delta \rightarrow 0$ appears explicitly in $I_{D}$, while $I_{R}$ is the integral of a regular function and does not diverge any more for $\delta \rightarrow 0$. Fig. 2 2 illustrates an interesting comparison between the original integrand of Eq. (26)

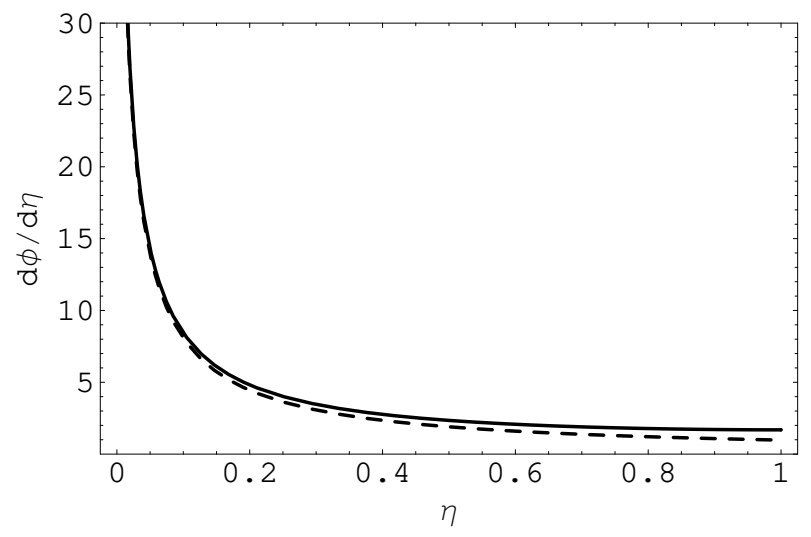

FIG. 2: The plot illustrates the integrand of $\Delta \phi_{i}$ in Eq. (26) as a function of $\eta$ for $\delta=0.025(\epsilon=0.001)$ in the case of Schwarzschild black hole; the dashed line is the integrand of $I_{D}$ in Eq. (28), the difference between the two curves being the integrand of $I_{R}$ in Eq. (29).

and the integrand of $I_{D}$ in Eq. (28) taking the special case of Schwarzschild metric as an example. Although the integrand of $I_{D}$ is a drastically simplified form of the original one which approximates it for $\eta$ very close to zero, it turns out to be a very good approximation in the whole range of $\eta$. Only for $\eta$ close to 1 we see a sensible difference. Such difference is stored in the integrand of $I_{R}$. As $\delta$ tends to zero, the contribution by $I_{D}$ becomes more and more dominant with respect to that of $I_{R}$ as the divergence of the integrand becomes stiffer and stiffer. These considerations are a good premise to the strong deflection limit.

Until now we have done no approximation. We have just added and subtracted some terms and made some changes of variables. The expression (27) is still exact. The strong deflection limit amounts to save the first dominant terms in the expressions for $I_{D}$ and $I_{R}$ as $\delta \rightarrow 0$. We have a logarithmic divergent term in $I_{D}$ and then some terms converging to constant values. We also note that the parametrization (23) tends to

$$
r=\frac{r_{m}}{1-\eta}
$$

when $\delta \rightarrow 0$. Consequently, the integration limits $\eta_{i}$ tend to $\eta_{i}=1-r_{m} / D_{L i}$.

After the truncation of the expansion in $\delta$, we have

$$
\Delta \phi_{i}=a \log \frac{2 \eta_{i}}{\delta}+b_{i}+o\left(\delta^{0}\right)
$$


where the coefficients $a$ and $b_{i}$ are given by

$$
\begin{aligned}
& a=r_{m} \sqrt{\frac{B_{m}}{A_{m} \beta_{m}}} \\
& b_{i}=\int_{0}^{\eta_{i}} g_{1}(\eta) d \eta=\int_{0}^{\eta_{i}} \operatorname{Sign}(\eta) g_{1}(\eta) d \eta \\
& g_{1}(\eta)=u_{m} \sqrt{\frac{B(\eta)}{C(\eta)}}\left[R\left(\eta, u_{m}\right)\right]^{-1 / 2} \frac{r_{m}}{(1-\eta)^{2}} \\
& -\frac{u_{m}}{\sqrt{\beta_{m}}} \sqrt{\frac{B_{m}}{C_{m}}} \frac{r_{m}}{|\eta|}
\end{aligned}
$$

where $g_{1}(\eta)$ is just the integrand of $I_{R}$ in the limit for $\delta \rightarrow 0$, also implying $u \rightarrow u_{m}$. The function $\operatorname{Sign}(\eta)$ has been introduced only for uniforming the expression of $b_{i}$ to the corresponding terms we shall derive in section IIB in the case of a source inside the photon sphere. For a source outside the photon sphere, the variable $\eta$ is always positive. Finally, the deflection suffered by the photon is quantified by the full azimuthal shift $\Delta \phi$, given by

$$
\Delta \phi=a \log \frac{4 \eta_{O} \eta_{S}}{\delta^{2}}+b_{O}+b_{S}
$$

When the source and the observer are very far from the black hole, it makes sense to define a deflection angle as the difference between the azimuthal shift suffered by the photon minus $\pi$, which represents the total azimuthal shift of a photon travelling in a flat space without the black hole on a rectilinear trajectory. This concept becomes ill-defined for sources and observers that are not in the asymptotic flat region.

The fact that source and observer are at finite distances is encoded in the presence of $\eta_{O}$ and $\eta_{S}$. Setting them to 1 , the deflection angle so derived coincides with the expression originally given in Ref. [23].

With the definition of $\beta_{m}$ given by Eq. (22) and Eq. (14), we can formulate an explicit expression for the coefficient of the logarithmic term in terms of the metric functions

$$
a=\sqrt{\frac{2 B_{m} A_{m}}{C_{m}^{\prime \prime} A_{m}-C_{m} A_{m}^{\prime \prime}}} .
$$

This coefficient is independent of the source and observer positions.

\section{B. Source inside the photon sphere}

Now let us consider the case in which the source is inside the photon sphere, but still outside the horizon. The photons leaving the source with negative $\dot{r}$ sink into the black hole. As for the photons leaving with positive $\dot{r}$, we have two possibilities: those starting with $u>$ $u_{m}$ meet an inversion point before reaching the photon sphere radius $r_{m}$. Therefore, they fall back into the black hole. The photons with $u<u_{m}$ meet no inversion point and escape towards the asymptotic region. The observer will thus see the deformed images of a source inside the photon sphere.

Recalling the relation between $u$ and the angle formed by the photon momentum with the tangential direction (Eq. (7)), we can re-interpret this discussion noting that only photons shot along the radial direction $(u=0)$ or very close to the radial direction $\left(u<u_{m}\right)$ will be able to escape to infinity. Photons shot at larger angles with respect to the radial direction invert their motion before crossing the photon sphere. It is interesting to note that the angle formed with the tangent direction by photons emitted at $D_{\mathrm{LS}}<r_{m}$ decreases until they cross the photon sphere. After that moment, they align more and more with the radial direction as they move farther and farther from the black hole.

At first sight, one may think that this situation is very different from the one described in the previous subsection. Actually, even in this case it is possible to define a strong deflection limit, corresponding to photons with $u$ just slightly smaller than $u_{m}$. Let us see this in detail.

The azimuthal shift of the photon is

$$
\begin{aligned}
\Delta \phi & =\int_{\phi_{i}}^{\phi_{f}} d \phi=\int_{D_{L S}}^{D_{O L}} \frac{\dot{\phi}}{\dot{r}} d r \\
& \int_{D_{L S}}^{D_{O L}} u \sqrt{\frac{B}{C}}[R(r, u)]^{-1 / 2} d r
\end{aligned}
$$

with $D_{L S}<r_{m}<D_{O L}$. Even if the function $R(r, u)$ never vanishes, it becomes minimum at $r=r_{m}$. Correspondingly, the integrand has a maximum at this point and is largely dominated by this peak at $r_{m}$ if $u$ is very close to $u_{m}$. So it is convenient to revisit the analysis of the function $R(r, u)$.

Now we have to keep in mind that $u<u_{m}$, so that the parametrization (19) yields $\epsilon<0$. As pointed before, the function $R(r, u)$ has no real roots when $u<u_{m}$, and in fact, Eq. (21) with $\epsilon<0$ gives an imaginary value for $\delta$, so that the inversion point $r_{0}=r_{m}(1+\delta)$ is no longer a real number. Moreover, it is again convenient to introduce the parametrization (31), but this time extended to $r<r_{m}$ corresponding to $\eta<0$. Thus now the $\eta$ variable ranges in the interval $1-\frac{r_{m}}{r_{S}}<\eta<1$.

We can now study the function $R(r(\eta), u)$ for small values of $\eta$ as in the previous subsection and find

$$
\begin{aligned}
& R(\eta, u) \simeq R\left(r_{m}, u_{m}\right)+\frac{\partial R}{\partial r}\left(r_{m}, u_{m}\right) r_{m}\left(\eta+\eta^{2}\right) \\
& +\frac{1}{2} \frac{\partial^{2} R}{\partial r^{2}}\left(r_{m}, u_{m}\right) r_{m}^{2} \eta^{2}-\frac{\partial R}{\partial u}\left(r_{m}, u_{m}\right) u_{m}|\epsilon| .
\end{aligned}
$$

As usual, the first two terms vanish because of Eqs. (15) and (16). As for the $\epsilon$-term, we can replace it by the corresponding $\delta^{2}$-term through Eq. (20). We just have 
to keep in mind that now $\delta^{2}<0$. The behavior of $R(\eta, u)$ for small $\eta$ is thus

$$
R(\eta, u)=\beta_{m}\left(-\delta^{2}+\eta^{2}\right)+o\left(\eta^{2}\right) .
$$

Returning to the integral (38), we can change the integration variable from $r$ to $\eta$ using Eq. (31) to get

$$
\Delta \phi=\int_{\eta_{S}}^{\eta_{O}} u \sqrt{\frac{B(\eta)}{C(\eta)}}[R(r, u)]^{-1 / 2} \frac{r_{m}}{(1-\eta)^{2}} d \eta
$$

where the integration extrema are already in the form $\eta_{i}=1-r_{m} / D_{L i}$. Note that, since the source is inside the photon sphere, we have $\eta_{S}<0$.

As before, we add and subtract a term that contains the main structure of the integrand, that is the peak at $\eta=0$, corresponding to $r=r_{m}$. We have

$$
\begin{aligned}
& \Delta \phi=I_{D}+I_{R} \\
& I_{D}=\int_{\eta_{S}}^{\eta_{O}} \frac{u_{m}}{\sqrt{\beta_{m}}} \sqrt{\frac{B_{m}}{C_{m}}} \frac{r_{m}}{\sqrt{-\delta^{2}+\eta^{2}}} d \eta \\
& I_{R}=\int_{\eta_{S}}^{\eta_{O}}\left[u \sqrt{\frac{B(\eta)}{C(\eta)}}[R(\eta, u)]^{-1 / 2} \frac{r_{m}}{(1-\eta)^{2}}\right. \\
& \left.-\frac{u_{m}}{\sqrt{\beta_{m}}} \sqrt{\frac{B_{m}}{C_{m}}} \frac{r_{m}}{\sqrt{-\delta^{2}+\eta^{2}}}\right] d \eta .
\end{aligned}
$$

The first integral is again elementary and reads

$$
I_{D}=r_{m} \sqrt{\frac{B_{m}}{A_{m} \beta_{m}}} \log \frac{\sqrt{-\delta^{2}+\eta_{O}^{2}}+\eta_{O}}{\sqrt{-\delta^{2}+\eta_{S}^{2}}-\left|\eta_{S}\right|},
$$

where we have made the sign of $\eta_{S}$ explicit. The second integral contains an integrand that is regular everywhere for $\delta \rightarrow 0$. Fig. 3 illustrates a comparison between the integrand of Eq. (41) and the integrand of $I_{D}$ in the Schwarzschild case. We see that even if there is no divergence for $\eta=0$, the integrand has a very pronounced peak that dominates the integral. The peak structure is catched by $I_{D}$, while the wings are corrected by the contribution of $I_{R}$. As $\epsilon \rightarrow 0$, the peak grows larger and larger, dominating the wings.

Now we make the strong deflection limit approximation, by requiring that $\epsilon$ and consequently $\delta^{2}$ is small. Saving the logarithmically divergent term and the constant terms, we have

$$
\Delta \phi=a \log \frac{4 \eta_{O} \eta_{S}}{\delta^{2}}+b_{S O}+o\left(\delta^{0}\right)
$$

where the coefficient $a$ is still given by Eq. (33) and $b_{S O}$ is

$$
b_{S O}=\int_{\eta_{S}}^{\eta_{O}} g_{1}(\eta) d \eta
$$

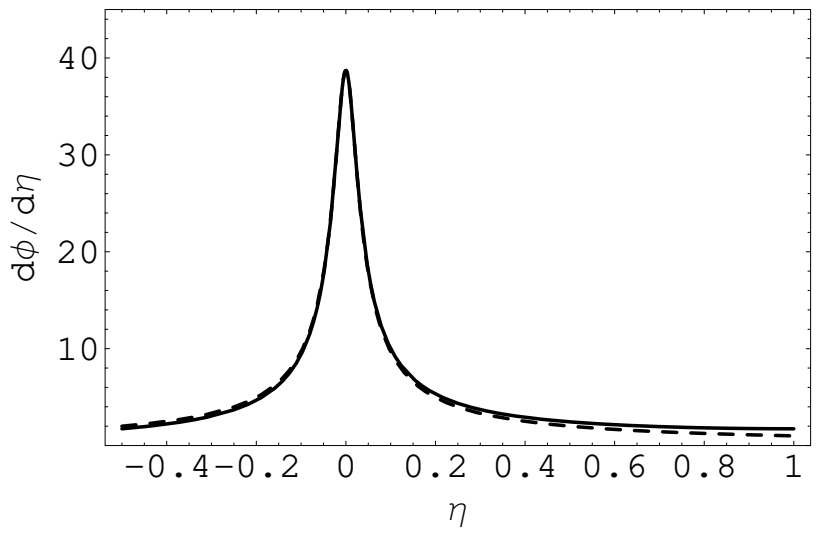

FIG. 3: The integrand of $\Delta \phi$ in Eq. (41) as a function of $\eta$ for $\delta=0.025 i(\epsilon=-0.001)$, compared to the integrand of $I_{D}$ in Eq. (43) in dashed style.

with $g_{1}(\eta)$ still given by Eq. (35). It is interesting to note that the argument of the logarithm remains positive, since both $\delta^{2}$ and $\eta_{S}$ are negative.

We can split the integral in Eq. (47) in two parts

$$
\begin{aligned}
& b_{S O}=\tilde{b}_{S}+\tilde{b}_{O} \\
& \tilde{b}_{S}=\int_{\eta_{S}}^{0} g_{1}(\eta) d \eta=\int_{0}^{\eta_{S}} \operatorname{Sign}(\eta) g_{1}(\eta) d \eta \\
& \tilde{b}_{O}=\int_{0}^{\eta_{O}} g_{1}(\eta) d \eta=\int_{0}^{\eta_{O}} \operatorname{Sign}(\eta) g_{1}(\eta) d \eta
\end{aligned}
$$

We observe that $\tilde{b}_{O}$ and $\tilde{b}_{S}$ have the same formal expression as $b_{O}$ and $b_{S}$.

\section{Deflection and higher order images formulas}

As a result of the previous two subsections, we have a unique expression for the photon deflection, which can be conveniently stated in terms of $\epsilon$, which represents the impact parameter shift from the minimum value (see Eq. (19)). By Eq. (36) and (21) we finally get

$$
\Delta \phi=-a \log \frac{\epsilon}{\eta_{O} \eta_{S}}+b+\pi,
$$

where we have defined the coefficient

$$
b=a \log \frac{2 \beta_{m}}{u_{m}^{2}}+b_{O}+b_{S}-\pi .
$$

For quick reference, $\epsilon$ is defined in Eq. (19), $u_{m}$ is given by Eq. (10), $\beta_{m}$ is given by Eq. (22), $a$ is given by Eq. (33), $\eta_{i}=1-r_{m} / D_{L i} ; b_{O}$ and $b_{S}$ are given by the integrals (34), where $g_{1}(\eta)$ is found in Eq. (35) and $R(r, u)$ in Eq. (14), having changed the integration variable from $r$ to $\eta$ through the parametrization (31). 
This expression for the total deflection of the photon is valid for any position of observer and source. Even if we have not explicitly considered it, time-reversal symmetry warrants that Eq. (51) is valid even in the unrealistic case of an observer inside the photon sphere (provided that one correctly relates $\epsilon$ to the sky coordinates of an observer in a strongly curved region). The only approximation performed is that the impact parameter $u$ is very close to the critical value $u_{m}$.

The general lens equation for spherically symmetric black holes can be simply stated as

$$
\phi_{O}-\phi_{S}=\Delta \phi \bmod 2 \pi
$$

Fixing the origin of the azimuthal coordinate in such a way that $\phi_{O}=\pi$ and using the expression of the total deflection in the strong deflection limit (51), we can easily solve the lens equation and find the position of the images. In general we have

$$
\epsilon_{n}=\eta_{O} \eta_{S} e^{\frac{b+\phi_{S}-2 n \pi}{a}}
$$

where $n$ denotes the number of loops done by the photons before reaching the observer and $\phi_{S} \in[-\pi, \pi]$. Of course, the strong deflection limit becomes exact in the limit $n \rightarrow$ $\infty$ but is typically a very good approximation already for $n=1$, as will be shown in the next subsection.

For an observer in the asymptotic region, which is the most physically interesting case, the angular separation between the direction of arrival of the photon and the direction of the black hole is simply $\theta=u / D_{O L}$. So, we have

$$
\begin{aligned}
& \theta=\theta_{m}(1+\epsilon) \\
& \theta_{m}=\frac{u_{m}}{D_{O L}} .
\end{aligned}
$$

$\theta_{m}$ is usually called the angular radius of the shadow of the black hole, since all images of sources outside the photon sphere reach the observer from angles $\theta>\theta_{m}$ and the region within the angular radius $\theta_{m}$ appears empty. However, when the source is inside the photon sphere, $D_{L S}<r_{m}$ and then $\eta_{S}<0$. We thus have $\epsilon<0$ and the sequence of images will appear within the shadow of the black hole, with the lowest order ones closer to the center and the higher order ones closer and closer to the shadow border.

The study of the Jacobian of the lens equation confirms that the critical curves are simply given by (55) and (54) with $\phi_{S}=0$ for standard lensing $\phi_{S}=\pi$ for retrolensing. They are circles outside the shadow for sources outside the photon sphere and inside the shadow for sources inside the photon sphere. The caustics are always pointlike and are located behind and in front of the source. Altogether, the caustics cover the whole optical axis as $D_{L S}$ varies from $+\infty$ to the static limit $r_{s}$.

It is interesting to take the limit of the total azimuthal shift $\Delta \phi$ for $D_{O L}, D_{L S} \gg r_{m}$ and calculate the first order in $r_{m} / D_{L i}$. Recalling that $\eta_{i}=1-r_{m} / D_{L i}$, we have

$$
\begin{aligned}
& \Delta \phi=-a \log \frac{u_{m}^{2} \epsilon}{2 \beta_{m}}-\frac{a r_{m}}{D_{O L}}-\frac{a r_{m}}{D_{L S}}+2 \int_{0}^{1} g(\eta) d \eta \\
& +g(1)\left(\eta_{O}-1\right)+g(1)\left(\eta_{S}-1\right)+o\left(\eta_{i}-1\right) .
\end{aligned}
$$

$g(1)$ can be calculated using the asymptotic limit of all metric functions evaluated in $\eta$ that appear in its expression and taking the limit for $\eta \rightarrow 1$. It is simply

$$
g(1)=\frac{u_{m}}{r_{m}}-a
$$

Summing up, we get

$$
\Delta \phi-\pi=\alpha-\theta_{m}-\bar{\theta}_{m}
$$

where $\alpha$ is the deflection angle calculated on the asymptotic trajectories $\left(D_{L S}=D_{O L}=\infty\right), \theta_{m}$ is defined in Eq. (56) and

$$
\bar{\theta}_{m}=\frac{u_{m}}{D_{L S}}
$$

is the angular size of the shadow of the black hole as measured by a distant source. The lens equation then becomes

$$
-\phi_{\mathrm{S}}=\alpha-\theta_{m}-\bar{\theta}_{m}
$$

The first correction to the lens equation with far source and observer is thus universal and simply takes into account the geometry of the lensing problem (compare with the discussion of the lens equation in Ref. [34]).

\section{Testing the formulas in the Schwarzschild case}

In this subsection, we shall specify all our general formulae for black hole gravitational lensing in the case of the simplest possible metric. This will allow us to understand the sense, the validity and the power of the strong deflection approximation throughout the range of possible source positions.

The Schwarzschild metric reads (with $G=c=1$ )

$$
\begin{aligned}
& A(r)=1-\frac{2 M}{r} \\
& B(r)=\left(1-\frac{2 M}{r}\right)^{-1} \\
& C(r)=r^{2} .
\end{aligned}
$$

The minimum of the function $C / A$ is at $r_{m}=3 M$. Correspondingly the minimum impact parameter $u_{m}=$ $\sqrt{C_{m} / A_{m}}=3 \sqrt{3} M$ as well-known [10, 33].

Now let us calculate the coefficients of the deflection formula in the strong deflection limit. As already noted after Eq. (37), the coefficient of the logarithmic term 
is independent of the source and observer positions. So, Eq. (37) simply gives the already known value

$$
a=1
$$

This can be expected, since the logarithmic term is a characteristic of geodesics winding around the photon sphere which does not depend on the start and arrival point.

The constant coefficient in the deflection formula is

$$
\begin{aligned}
& b=-\pi+5 \log [6]+b_{O}+b_{S} \\
& b_{i}=-2 \log \left[3+\sqrt{3+\frac{18 M}{D_{L i}}}\right] .
\end{aligned}
$$

Putting everything together, we have

$$
\Delta \phi-\pi=-\log \frac{\epsilon}{\left(1-3 M / D_{L S}\right)\left(1-3 M / D_{O L}\right)}+b,
$$

which reduces to the well-known formula [10]

$$
\alpha=-\log \epsilon+\log \left[216(2-\sqrt{3})^{2}\right]-\pi
$$

in the limit $D_{O L}, D_{L S} \rightarrow \infty$. Eq. (68) is the generalization of Darwin's formula (69) to sources and observers at finite distance from the black hole, the only approximation remaining $|\epsilon| \ll 1$ (see Appendix $\mathrm{A}$ for a discussion of formulae expressed in terms of alternative perturbative parameters). In order to test our formula for the deflection of a photon, we can use it to calculate the radius of the critical curves.

The angular radius of the critical rings is given by Eq. (54) with $\phi_{S}=0$ or $\pi$. Explicitly, for the Schwarzschild metric we have

$$
\begin{aligned}
& \theta_{k}=\frac{3 \sqrt{3} M}{D_{O L}}\left(1+\epsilon_{k}\right) \\
& \epsilon_{k}=\frac{216(2-\sqrt{3})\left(2 D_{L S}-3\right)}{\left(\sqrt{3 D_{L S}}+\sqrt{3+D_{L S}}\right)^{2}} e^{-k \pi},
\end{aligned}
$$

where $k$ is an even number in the retro-lensing case $\left(\phi_{S}=\pi\right)$, and an odd number in the standard lensing case $\left(\phi_{S}=0\right)$. The first critical curve for $k=1$ is created by photons experiencing weak deflection and is beyond the range of validity of Eq. (70). The critical curve with $k=2$ is the first retro-lensing ring, while for $k=3$ we obtain the first higher order Einstein ring of standard lensing.

The displacement of the first relativistic Einstein ring from the black hole shadow is shown in Fig. 4h, where it can be appreciated how $\epsilon_{3}$ stays small throughout the range of source distances, validating the strong deflection limit approximation. For $D_{L S} \gg M$, the ring radius tends to its asymptotic value. Our analytic formula for the critical curve nicely joins the region within the photon sphere $D_{L S}<3 M$ to that outside the photon sphere $D_{L S}>3 M$. The divergent term in the deflection formula at $D_{L S}=3 M$ forces $\epsilon=0$, in order to keep $\Delta \phi$ finite.
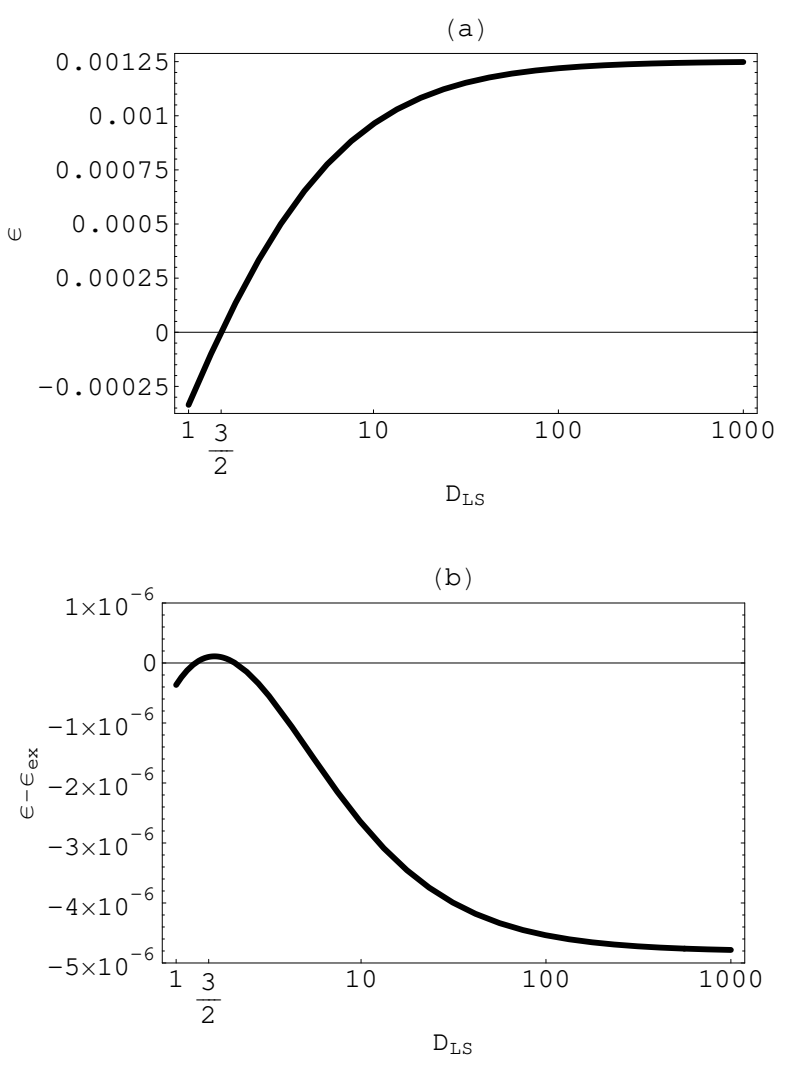

FIG. 4: (a) Angular radius of the first relativistic Einstein ring relative to the black hole shadow for a source behind the black hole as a function of the source distance measured in Schwarzschild radii $\left(\epsilon_{3}=\left(\theta_{3}-\theta_{m}\right) / \theta_{m}, 2 M=1\right)$. (b) Difference between the value of $\epsilon_{3}$ calculated by the strong deflection limit formula and the one calculated by the exact formula.

Since this is true for any order $k$, we can conclude that all higher order images of a source right at the photon sphere collapse into one degenerate image appearing right at the shadow border. As $D_{L S}<3 M, \epsilon$ becomes negative as expected. This means that the sequence of Einstein rings for a source inside the photon sphere is reversed: the brightest rings will appear closer to the center of the shadow and the fainter will be farther, approaching the shadow border as $k$ grows more and more.

The Schwarzschild metric is simple enough to allow an exact integration of the azimuthal motion in terms of elliptic integrals. It is thus very important and instructive to compare the critical curve radius calculated by the formula (51) obtained in the strong deflection limit to the exact Einstein ring position, calculated using the exact deflection angle. The difference between the two positions is plotted in Fig. 4b, where it can be appreciated that it stays of the order $\epsilon^{2}$ throughout the range of $D_{L S}$, testifying the accuracy of the strong deflection limit as a powerful approximation to describe higher order images.

The updated analysis of extreme gravitational lensing 
by Schwarzschild black holes presented here can be repeated for any kind of spherically symmetric black holes, using the formulae derived in this section. It is interesting to consider that some metrics could give the same lensing observables for sources at infinity, whereas they could be distinguished in gravitational lensing of sources at small distances from the black hole. Therefore, the introduction of $D_{L S}$ as a new parameter enriches the arena for the comparison of different metrics.

\section{KERR BLACK HOLE}

In this section, we shall describe gravitational lensing of sources placed at arbitrary distances from a spinning black hole. With respect to the analysis of Ref. [22], the finiteness of $D_{O L}$ and $D_{L S}$ only intervenes in the calculation of the radial integrals. As a consequence, the first three sections of Ref. [22], concerning the description of the unstable circular orbit and the shadow of the Kerr black hole, remain unaffected. Their content is briefly reviewed in the following subsection. The modifications in the radial integrals are reflected in the lens equation and its Jacobian. Consequently, also the critical curves and the caustics contain a dependence on the source position. They are described in Section IIIB. Finally, lensing of sources near caustics is updated in Section IIIC. Throughout this section, we shall preserve the spirit of Ref. [22] expanding all quantities to the second order in the black hole spin $a$. The perturbative expansion is of key-importance to keep all calculations fully analytic up to the final results. At the same time, the second order approximation proves to be very reliable up to values of the black hole spin $a \simeq 0.1$, as noted in the comparison with numerical results 21].

\section{A. Derivation of the lens equation}

The Kerr metric in Boyer-Lindquist coordinates [36] is

$$
\begin{aligned}
d s^{2}= & \frac{\Delta-a^{2} \sin ^{2} \vartheta}{\rho^{2}} d t^{2}-\frac{\rho^{2}}{\Delta} d r^{2}-\rho^{2} d \vartheta^{2} \\
& -\frac{\left(r^{2}+a^{2}\right)^{2}-a^{2} \Delta \sin ^{2} \vartheta}{\rho^{2}} \sin ^{2} \vartheta d \phi^{2} \\
& +\frac{2 a r \sin ^{2} \vartheta}{\rho^{2}} d t d \phi \\
\Delta= & r^{2}-r+a^{2}, \\
\rho^{2}= & r^{2}+a^{2} \cos ^{2} \vartheta .
\end{aligned}
$$

Distances are measured in units of the Schwarzschild radius $\left(2 M G / c^{2}=1\right)$ and $a$ is the specific angular momentum of the black hole, running from 0 (Schwarzschild black hole) to $1 / 2$ (extremal Kerr black hole) in our units.

The Kerr geodesics are described in integral form by the equations

$$
\begin{aligned}
& \pm \int \frac{d r}{\sqrt{R}}= \pm \int \frac{d \vartheta}{\sqrt{\Theta}} \\
\phi_{O}-\phi_{S}= & a \int \frac{r^{2}+a^{2}-a J}{\Delta \sqrt{R}} d r-a \int \frac{d r}{\sqrt{R}} \\
& +J \int \frac{\csc ^{2} \vartheta}{\sqrt{\Theta}} d \vartheta,
\end{aligned}
$$

with

$$
\begin{aligned}
\Theta= & Q+a^{2} \cos ^{2} \vartheta-J^{2} \cot ^{2} \vartheta \\
R= & r^{4}+\left(a^{2}-J^{2}-Q\right) r^{2}+\left(Q+(J-a)^{2}\right) r \\
& -a^{2} Q .
\end{aligned}
$$

$J$ is the component of the angular momentum of the photon along the spin axis and $Q$ is the Carter integral [2], related to the total angular momentum of the photon (we set the specific energy $E$ to 1 by a suitable choice of the affine parameter).

An observer in the position $\left(D_{O L}, \vartheta_{O}, \phi_{O}\right)$, defines angular coordinates $\left(\theta_{1}, \theta_{2}\right)$ in the sky, such that the black hole is in $(0,0)$ with its spin projected along $\theta_{2}$. A photon travelling on a geodesic characterized by constants of motion $J$ and $Q$ hits a distant observer from the direction

$$
\begin{aligned}
& \theta_{1}=-\frac{J}{D_{O L} \sqrt{1-\mu_{O}^{2}}} \\
& \theta_{2}= \pm D_{O L}^{-1} \sqrt{Q+\mu_{O}^{2}\left(a^{2}-\frac{J^{2}}{1-\mu_{O}^{2}}\right)}
\end{aligned}
$$

where $\mu_{O} \equiv \cos \vartheta_{O}$ as usual [33].

\section{Shadow of the Kerr black hole}

Among all photon trajectories ending at the observer, there is a family of trajectories that approach an unstable circular orbit around the black hole when traced back asymptotically in the past. This family can be parameterized by the parameter $\xi$ ranging from -1 to 1 . The constants of motion identifying the geodesics of this family are then given by

$$
\begin{aligned}
J_{m}(\xi)= & \frac{3 \sqrt{3}}{2} \xi \sqrt{1-\mu_{O}^{2}}-a\left(1-\mu_{O}^{2}\right)\left(1+\xi^{2}\right) \\
& -a^{2} \frac{\xi \sqrt{1-\mu_{O}^{2}}}{3 \sqrt{3}}\left[5-2 \xi^{2}-2 \mu_{O}^{2}\left(1-\xi^{2}\right)\right](81) \\
Q_{m}(\xi)= & \frac{27}{4}\left[1-\left(1-\mu_{O}^{2}\right) \xi^{2}\right] \\
& -3 \sqrt{3} a \xi \sqrt{1-\mu_{O}^{2}}\left[1+\mu_{O}^{2}-\left(1-\mu_{O}^{2}\right) \xi^{2}\right] \\
& -a^{2}\left[\left(1+\mu_{O}^{2}\right)^{2}-4\left(1-\mu_{O}^{2}\right) \xi^{2}\right. \\
& \left.+3\left(1-\mu_{O}^{2}\right)^{2} \xi^{4}\right] .
\end{aligned}
$$


The radius of the unstable circular orbit asymptotically approached in the past is

$$
\begin{aligned}
r_{m}= & \frac{3}{2}-\frac{2}{\sqrt{3}} a \xi \sqrt{1-\mu_{O}^{2}}-\frac{4}{9} a^{2}\left(1+\mu_{O}^{2}\right) \\
& -\frac{4}{27 \sqrt{3}} a^{3} \xi\left(5+6 \mu_{O}^{2}\right) \sqrt{1-\mu_{O}^{2}}+O\left(a^{4}\right) .
\end{aligned}
$$

Correspondingly, a distant observer sees such photons from the directions

$$
\begin{aligned}
& D_{O L} \theta_{1, m}=-\frac{3 \sqrt{3}}{2} \xi+a \sqrt{1-\mu_{O}^{2}}\left(1+\xi^{2}\right) \\
& +a^{2} \frac{\xi}{3 \sqrt{3}}\left[5-2 \mu_{O}^{2}-2 \xi^{2}\left(1-\mu_{O}^{2}\right)\right] \\
& D_{O L} \theta_{2, m}= \pm \frac{3 \sqrt{3}}{2} \sqrt{1-\xi^{2}} \mp a \xi \sqrt{1-\xi^{2}} \sqrt{1-\mu_{O}^{2}} \\
& \mp a^{2} \frac{\sqrt{1-\xi^{2}}}{3 \sqrt{3}}\left[1+2 \mu_{O}^{2}-2 \xi^{2}\left(1-\mu_{O}^{2}\right)\right] \text {. }
\end{aligned}
$$

Eqs. (84) and (85) define a curve in the observer sky as $\xi$ varies between -1 and 1 . This curve represents the border of the shadow of the black hole, in the sense that all photons emitted by a source outside the unstable circular orbits reach the observer from directions outside this border. In the Schwarzschild limit, the shadow border is simply a circle of radius $3 \sqrt{3} / 2 D_{O L}$, whereas for generic values of the spin, the shadow satisfies the ellipse equation

$$
\frac{\left(\theta_{1, m}-\theta_{0}\right)^{2}}{A_{1}^{2}}+\frac{\theta_{2, m}^{2}}{A_{2}^{2}}=1+o\left(a^{2}\right)
$$

with the origin shifted rightward by

$$
\theta_{0}=\frac{2 a \sqrt{1-\mu_{o}^{2}}}{D_{O L}}
$$

and semiaxes given by

$$
\begin{aligned}
& A_{1}=\frac{3 \sqrt{3}}{2 D_{O L}}\left(1-\frac{2}{9} a^{2}\right) \\
& A_{2}=\frac{3 \sqrt{3}}{2 D_{O L}}\left(\frac{3 \sqrt{3}}{2}-\frac{2 \mu_{o}^{2}}{9} a^{2}\right),
\end{aligned}
$$

with ellipticity

$$
e \equiv 1-\frac{A_{1}}{A_{2}}=\frac{2}{9} a^{2}\left(1-\mu_{o}^{2}\right)
$$

For more details on the derivation of the parameterization (83) and of the shadow border, see the deep and detailed discussion in Ref. 22].

\section{Strongly deflected photons}

We now introduce the following parametrization of the observer sky by

$$
\left\{\begin{array}{l}
\theta_{1}(\epsilon, \xi)=\theta_{1, m}(\xi)(1+\epsilon) \\
\theta_{2}(\epsilon, \xi)=\theta_{2, m}(\xi)(1+\epsilon)
\end{array}\right. \text {. }
$$

One half of the sky is covered as $\xi$ varies from -1 to 1 and $\epsilon$ varies from -1 to $+\infty$. The double sign in $\theta_{2, m}$ selects which half of the sky we are covering. We are interested into strongly deflected photons, which correspond to very small values of $\epsilon$. One may regard $\epsilon$ as the relative displacement of the photon direction from the shadow border. As $\epsilon \rightarrow 0$, the photon spends more and more time close to the unstable circular orbit, and performs more and more loops around the black hole before emerging.

Using Eqs. (79) and (80) we can calculate the values of $J$ and $Q$ identifying the geodesics of such photons. For each value of $J$ and $Q$, we can calculate the inversion point $r_{0}$ in the radial motion examining the roots of the function $R(r, J, Q)$, defined by Eq. (78). It is immediate to see that $R(r, J, Q)$ satisfies the properties

$$
\begin{aligned}
& \frac{\partial R}{\partial r}\left(r_{m}, J_{m}, Q_{m}\right)=0 \\
& R\left(r_{m}, J_{m}, Q_{m}\right)=0 \\
& R\left(r_{0}, J, Q\right)=0 .
\end{aligned}
$$

Analogously to the spherically symmetric case, we can define the parameter $\delta$ by

$$
r_{0}=r_{m}(1+\delta)
$$

$\delta$ is thus the relative displacement of the inversion point from the unstable circular orbit radius. Inserting the expression of $J$ and $Q$ as functions of $\xi$ and $\epsilon$ and solving Eq. (94) for $\delta$ to the lowest order in $\epsilon$ we find that the two parameters are related by

$$
\delta=\sqrt{\frac{2 \epsilon}{3}}\left[1-\frac{2}{3 \sqrt{3}} a \hat{\xi}+\frac{2}{27} a^{2}\left(10-\mu_{o}^{2}-14 \hat{\xi}^{2}\right)\right],
$$

where we have introduced the compact notation

$$
\hat{\xi}=\xi \sqrt{1-\mu_{o}^{2}}
$$

So, for any photons hitting the observer from a direction close to the shadow border, we can immediately find the corresponding value of the inversion point in its motion around the black hole. If the photon comes from the interior of the shadow $(\epsilon<0)$, then the inversion point becomes complex, signaling the fact that the photon follows a geodesic without inversion points in the radial motion. 


\section{From the geodesics equation to the lens equation}

Now we can come to the resolution of the radial integrals appearing in the geodesics equations (75) and (76). We indicate them by

$$
\begin{aligned}
& I_{1}= \pm \int \frac{d r}{\sqrt{R}} \\
& I_{2}= \pm \int \frac{r^{2}+a^{2}-a J}{\Delta \sqrt{R}} d r .
\end{aligned}
$$

These integrals must be performed along the photon path from the source to the observer. The double sign indicates that one has to sum the contributions with different signs of $\dot{r}$ coherently. The task is simplified by the observation that the function $R(r, J, Q)$ determining the radial motion in the Kerr metric satisfies the same properties as the function $R(r, u)$ determining the radial motion in spherically symmetric metrics (compare Eqs. (92)-(94) to Eqs. (15)-(17)). So, we can repeat exactly the same steps of the analysis of the function $R(r, u)$, with the trivial extension to the presence of two constants of motion. The integrals are then easily solved to

$$
\begin{aligned}
& I_{1}=-a_{1} \log \delta+b_{1}+c_{1}\left(D_{L S}\right)+c_{1}\left(D_{O L}\right) \\
& I_{2}=-a_{2} \log \delta+b_{2}+c_{2}\left(D_{L S}\right)+c_{2}\left(D_{O L}\right),
\end{aligned}
$$

where the coefficients $a_{1}, b_{1}, a_{2}$, and $b_{2}$ remain the same as those given in the appendix of Ref. [22], whereas the explicit expressions of the new functions $c_{1}$ and $c_{2}$ are shown in the Appendix B at the end of this work (Eqs. (B3) and (B4) $)$. We have stored the whole dependence on the source and observer distance in these two coefficients. They are defined in such a way that they vanish when their argument goes to infinity, so that the expressions for distant sources and observers are recovered. Although we have provided the expressions of the integrals for arbitrary observer distances, we shall consider $D_{O L} \gg 1$ from now on for simplicity. If one is interested to study gravitational lensing with observers close to the black hole, it is easy to recover the relevant formulae, since the dependence on $D_{L S}$ and $D_{O L}$ is interchangeable, thanks to the symmetry $D_{L S} \leftrightarrow D_{O L}$ in Eqs. (100) and (101).

In the angular integrals

$$
\begin{aligned}
& J_{1}= \pm \int \frac{1}{\sqrt{\Theta}} d \vartheta \\
& J_{2}= \pm \int \frac{\csc ^{2} \vartheta}{\sqrt{\Theta}} d \vartheta
\end{aligned}
$$

there is no dependence on the source and observer distance. Therefore, we can safely exploit the expressions in the appendix of Ref. [22] without any more concern.

Finally, it is convenient to replace the parameter $\delta$ (which, as we recall, represents the relative displacement of the inversion point from the radius of the unstable circular orbit) by a new variable

$$
\psi=-2 \log \delta+\log \left[\frac{144(7-4 \sqrt{3})\left(2 D_{L S}-3\right)}{\left(\sqrt{3 D_{L S}}+\sqrt{3+D_{L S}}\right)^{2}}\right] .
$$

From the physical point of view, $\psi$ simply represents the equivalent azimuthal shift of a photon deflected by a Schwarzschild black hole.

Once all integrals are solved, we can rearrange the integrated geodesics equations in the typical form of a lens equation, moving the source angular coordinates on the left hand side and leaving everything else on the right hand side

$$
\left\{\begin{array}{l}
\mu_{S}=\mu_{S}\left(\psi, \xi ; \mu_{O}, D_{O L}, D_{L S}\right) \\
\phi_{S}=\phi_{S}\left(\psi, \xi ; \mu_{O}, D_{O L}, D_{L S}\right)
\end{array} .\right.
$$

The lens mapping relates the source coordinates $\left(\mu_{S}, \phi_{S}\right)$ to the set of intermediate variables $(\psi, \xi)$, which characterize the photon geodesic by the amount of deflection and its orientation in space respectively. The sky coordinates $\left(\theta_{1}, \theta_{2}\right)$ are related to $(\psi, \xi)$ by Eqs. (91), (96) and (104). The observer position and the source distance play the role of parameters of the lens mapping.

In the following subsections we shall put in evidence the main features of the lens mapping through the analysis of its critical points and the discussion of gravitational lensing near the critical points.

\section{B. Critical curves and caustics}

In order to find the critical points of the lens equation, one has to calculate the Jacobian determinant

$$
\operatorname{det} J=\frac{\partial \mu_{s}}{\partial \xi} \frac{\partial \phi_{s}}{\partial \psi}-\frac{\partial \mu_{s}}{\partial \psi} \frac{\partial \phi_{s}}{\partial \xi} .
$$

The critical points of the lens mapping are the solutions of the equation $\operatorname{det} J(\psi, \xi)=0$. The critical curves are the corresponding points in the observer sky $\left(\theta_{1}, \theta_{2}\right)$ through Eq. (91) and the caustics are the images of these points in the $\left(\mu_{s}, \phi_{s}\right)$ space through the lens mapping. The full procedure is straightforward and is detailed in Ref. 22]. Here we just state the updated results with the encompassment of the finiteness of $D_{L S}$.

\section{Critical points in the $(\psi, \xi)$ space}

As mentioned before, $\psi$ represents the equivalent azimuthal shift of a photon moving in the Schwarzschild metric obtained turning the black hole spin off. It is thus not surprising that the zero-order critical points are simply given by $\psi_{k}^{(0)}=k \pi$, with $k$ being an integer number. $k$ is the number of half-loops performed by a photon in the Schwarzschild metric. We will often refer to this integer number as the critical curve order or caustic order. $k=1$ gives the azimuthal shift of a photon emitted by a source behind the black hole and weakly deflected by the black hole. Such photons are not the subject of our analysis. The first interesting case is $k=2$ corresponding to photons emitted by a source in front of the black hole and backscattered by the black hole (retro-lensing). 
Photons with $k=3$ are again emitted by a source behind the black hole, but now the photons perform a complete loop around the black hole before reaching the observer. Summing up, odd critical orders are involved in gravitational lensing of a source behind the black hole, whereas even critical orders are involved in retro-lensing. For each $k$ we have a different critical curve, which physically corresponds to the degenerate image of a source placed on the corresponding caustic. Starting by the zero-order solution, the first and second order correction can be found solving the Jacobian equation order by order. For each $k$, we have

$$
\psi_{k}(\xi)=k \pi+a \psi_{k}^{(1)}(\xi)+a^{2} \psi_{k}^{(2)}(\xi),
$$

with $\psi_{k}^{(1)}$ and $\psi_{k}^{(2)}$ given by

$$
\begin{aligned}
\psi_{k}^{(1)} & =\frac{2 \hat{\xi} \sqrt{D_{L S}}}{\left(2 D_{L S}-3\right) \sqrt{3+D_{L S}}} \\
\psi_{k}^{(2)} & =-\frac{1}{18}\left[9 c_{k}\left(3-2 \mu_{O}^{2}-3 \hat{\xi}^{2}\right)+32\left(1-\hat{\xi}^{2}\right)\right] \\
& -\left[3 \sqrt{3}\left(2 D_{L S}-3\right)^{2} \sqrt{D_{L S}}\left(3+D_{L S}\right)^{3 / 2}\right]^{-1} \\
& \cdot\left\{( 2 D _ { L S } - 3 ) ( 3 + D _ { L S } ) \left[2\left(\sqrt{D_{L S}}-\sqrt{3+D_{L S}}\right)^{2}\right.\right. \\
& \cdot\left(2 D_{L S}-3\right)\left(3-2 \mu_{O}^{2}-3 \hat{\xi}^{2}\right) \\
& \left.+26 D_{L S}-27-\left(4 D_{L S}-18\right) \mu_{O}^{2}-\left(18 D_{L S}-27\right) \hat{\xi}^{2}\right] \\
& \left.-108 D_{L S} \hat{\xi}^{2}\right\} .
\end{aligned}
$$

In the limit $D_{L S} \rightarrow \infty$ the first order correction vanishes while the second order correction reduces to the first row. These correctly reproduce the results of Ref. 22]. As $\xi$ varies between -1 and 1 , we recover all critical points in the $(\psi, \xi)$ space.

\section{Critical curves}

Starting from the solution of the Jacobian determinant equation in the $(\psi, \xi)$ space, we can construct both the critical curves and the caustics. As for the critical curves, it is sufficient to use Eq. (91) with $\epsilon$ expressed by Eqs. (96) and (104) in terms of $\psi$ and put $\psi=\psi_{k}$ as given by Eq. (107). In this way one gets the critical curves in the form $\left(\theta_{1, k}(\xi), \theta_{2, k}(\xi)\right)$. These expressions are lengthy and are not very transparent. However, it is straightforward to prove that they satisfy the ellipse equation at the second order in $a$

$$
\frac{\left(\theta_{1, k}-\theta_{0, k}\right)^{2}}{A_{1, k}^{2}}+\frac{\theta_{2, k}^{2}}{A_{1, k}^{2}}=1+o\left(a^{2}\right) .
$$

The center of the critical curve is shifted by the quantity

$$
D_{O L} \theta_{0, k}=2 a \sqrt{1-\mu_{o}^{2}}\left(1-\frac{3 \sqrt{3 D_{L S}} \epsilon_{k}}{\left(2 D_{L S}-3\right) \sqrt{3+D_{L S}}}\right)
$$

which reduces to the shadow shift in the limit $D_{L S} \rightarrow \infty$. For generic values of the source distance, the center of the critical curve does not coincide with the center of the shadow. However, the displacement is very small, since it is proportional to $\epsilon_{k}$ (given in Eq. (71), which is very small in the strong deflection limit. We can also note that the degeneracy between the black hole spin $a$ and the observer position $\sqrt{1-\mu_{o}^{2}}$ that was pointed out in Ref. 22] holds even when the source is at finite distance. Fig. 5 a shows the dependence of the shift with $D_{L S}$. For any value of the critical order $k$, the shift tends to the shadow shift for large values of $D_{L S}$. The first retrolensing critical curve $(k=2)$ is the most displaced one.

The major semiaxis of the critical curve is oriented along the projection of the spin on the observer sky. Its explicit expression is

$$
\begin{aligned}
A_{2, k} & =\frac{3 \sqrt{3}}{2 D_{O L}}+\frac{a^{2}}{D_{O L}}\left\{-\frac{\mu_{O}^{2}}{\sqrt{3}}+\epsilon_{k} \frac{4 \mu_{O}^{2}-9 c_{k}\left(2 \mu_{O}^{2}-3\right)}{4 \sqrt{3}}\right. \\
& +\epsilon_{k}\left[2 \sqrt{D_{L S}} \sqrt{3+D_{L S}}\left(2 D_{L S}-3\right)\right]^{-1} \\
& {\left[2\left(2 D_{L S}-3\right)\left(\sqrt{D_{L S}}-\sqrt{3+D_{L S}}\right)^{2}\left(3-2 \mu_{O}^{2}\right)\right.} \\
& \left.\left.+9\left(2 D_{L S}-3\right)+2 \mu_{O}^{2}\left(2 D_{L S}+9\right)\right]\right\},
\end{aligned}
$$

and is plotted in Fig. $5 \mathrm{~b}$ as a function of the source distance. We can appreciate that all critical curves are external to the shadow as $D_{L S}>3 / 2$ and internal to it when $D_{L S}<3 / 2$, as in the spherically symmetric case. The larger the order of the critical curve, the closer the curve is to the shadow, as $\epsilon_{k} \rightarrow 0$ for $k \rightarrow \infty$.

Rather than giving the expression of the minor semiaxis, it is instructive to examine the ellipticity of the critical curve, defined by

$$
e_{k}=1-\frac{A_{1, k}}{A_{2, k}}
$$

Of course, $A_{1, k}$ can be easily derived by this expression if one is interested in it. To the second order in $a$ and to the first order in $\epsilon_{k}$, the ellipticity is

$$
\begin{aligned}
e_{k} & =a^{2}\left(1-\mu_{O}^{2}\right)\left\{\frac{2}{9}+\frac{\left(27 c_{k}-8\right) \epsilon_{k}}{18}\right. \\
& -\frac{4 \epsilon_{k}}{\sqrt{3}}+\epsilon_{k}\left[9 \sqrt{D_{L S}}\left(3+D_{L S}\right)^{3 / 2}\left(2 D_{L S}-3\right)^{2}\right]^{-1} \\
& \cdot\left[\sqrt{3}\left(729-225 D_{L S}-348 D_{L S}^{2}+92 D_{L S}^{3}+48 D_{L S}^{4}\right)\right. \\
& \left.\left.-72 D_{L S}^{3 / 2} \sqrt{3+D_{L S}}\right]\right\} .
\end{aligned}
$$

This quantity reduces to the first row when $D_{L S} \rightarrow \infty$, which is the same as that given in Ref. [22] safe for the fact that we stop here at the first order in $\epsilon_{k}$ in order to be consistent with the strong deflection limit approximation. For large values of $k, \epsilon_{k} \rightarrow 0$ and the ellipticity of the critical curves tend to the ellipticity of the shadow (90). Fig. 

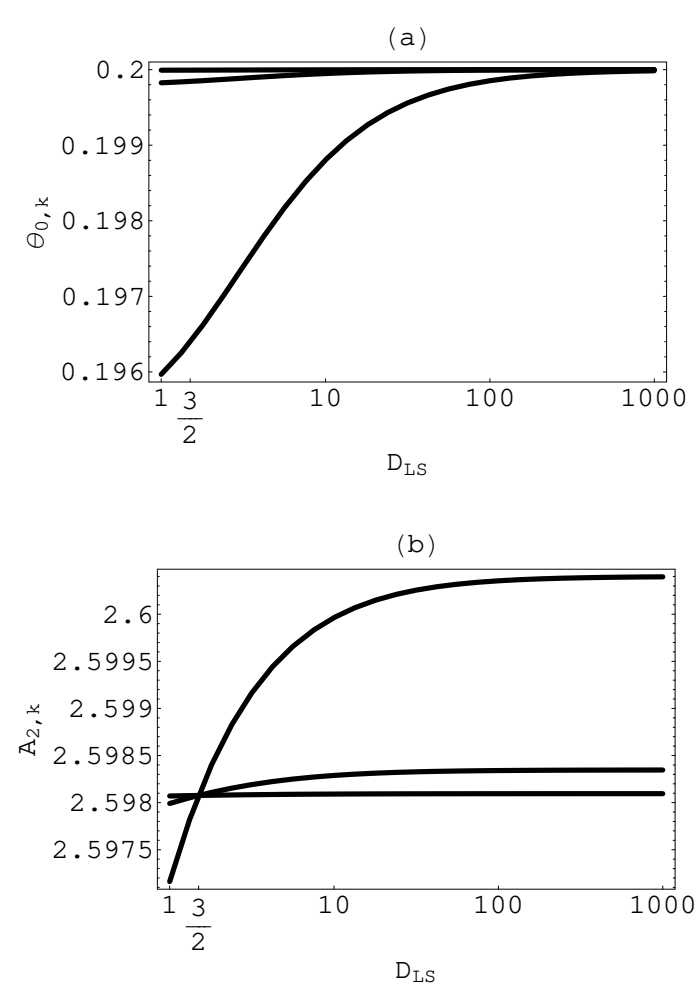

(c)

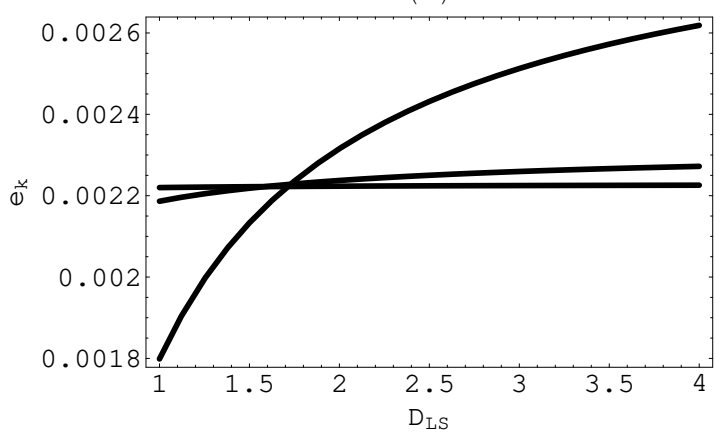

FIG. 5: (a) Apparent shift of the center of the critical curves with respect to the black hole position as a function of the source distance; from the bottom up, the curves are for $k=2,3,4$, respectively. (b) Major semiaxis of the critical curves with $k=2,3,4$ (from top to bottom) as a function of the source distance. (c) Ellipticity of the critical curves as a function of the source distance; from the top down the curves are done for $k=2,3,4$, respectively. All plots are done for $a=0.1$ and equatorial observer $\mu_{O}=0$.

5: shows the ellipticity of the first three relativistic critical curves as functions of the source distance. We note that all curves are more elliptical than the shadow when $D_{L S}$ is large. But for sources just slightly farther than the unstable circular photon orbit, the ellipticity of the critical curves equals the ellipticity of the shadow. This happens at a value of $D_{L S}$ slightly greater than $3 / 2$ and different for all critical curves. Below this distance, the ellipticity of the critical curves becomes smaller than that of the shadow. Finally, we can note that the ellipticity remains a function of $a \sqrt{1-\mu_{O}^{2}}$, thus preserving the degeneracy between the black hole spin and its orientation relative to the line of sight.

\section{Caustics}

The caustics are obtained evaluating the lens mapping in the critical points $\psi_{k}$ given by Eq. (107). To the zero order, the Schwarzschild caustics are recovered. They are pointlike and placed behind the black hole for $k$ odd and in front of the black hole for $k$ even. As the black hole spin is turned on, the caustics drift from the optical axis and acquire a finite extension. Their explicit expression is

$$
\begin{aligned}
& \mu_{S}=(-1)^{k} \mu_{O} \pm R_{k} \sqrt{1-\mu_{O}^{2}}\left(1-\xi^{2}\right)^{3 / 2}, \\
& \phi_{S}=(1-k) \pi-\Delta \phi_{k}-\frac{R_{k}}{\sqrt{1-\mu_{o}^{2}}} \xi^{3},
\end{aligned}
$$

with the drift given by

$$
\begin{aligned}
\Delta \phi_{k} & =a\left\{\frac{4 k \pi}{3 \sqrt{3}}+2 \log \left[3(2-\sqrt{3})^{2}\right]\right. \\
& \left.+\log \left[\frac{\left(2 \sqrt{D_{L S}}+\sqrt{3+D_{L S}}\right)^{2}}{9\left(D_{L S}-1\right)}\right]\right\},
\end{aligned}
$$

and the semi-amplitude given by

$$
\begin{aligned}
R_{k} & =a^{2}\left(1-\mu_{O}^{2}\right)\left[c_{k}\right. \\
& \left.+\frac{2\left(9+4 D_{L S}-4 \sqrt{D_{L S}} \sqrt{3+D_{L S}}\right)}{3 \sqrt{3} \sqrt{D_{L S}} \sqrt{3+D_{L S}}}\right]
\end{aligned}
$$

The caustic is a four-cusped astroid with the same angular extension along both axes, as can be explicitly seen transforming the above expressions to coordinates centered on the caustic (see Ref. [22]). The outstanding feature that emerges from these expressions is that the drift of the caustic diverges logarithmically as the source approaches the horizon. As can be seen in Fig. 6a, the drift is always negative (clockwise as seen from the northern pole of the black hole) and grows linearly with the caustic order $k$. The drift tends to the asymptotic value (given by the first row of Eq. (117)) for large values of $D_{L S}$, while it grows monothonically as $D_{L S}$ is decreased.

The amplitude of the caustic does not present any divergences. As shown in Fig. 6b, the amplitude increases linearly with the caustic order and tends to the asymptotic value (given by the first row in Eq. (118) for large $D_{L S}$. As $D_{L S}$ is decreased up to the horizon it grows by a fixed amount, given by

$$
R_{k}(1)-R_{k}(\infty)=\frac{5}{3 \sqrt{3}} a^{2}\left(1-\mu_{O}^{2}\right) .
$$

Taking $D_{L S}$ as a parameter ranging from 1 to $+\infty$, we can trace the whole caustic hypersurface using Eqs. 

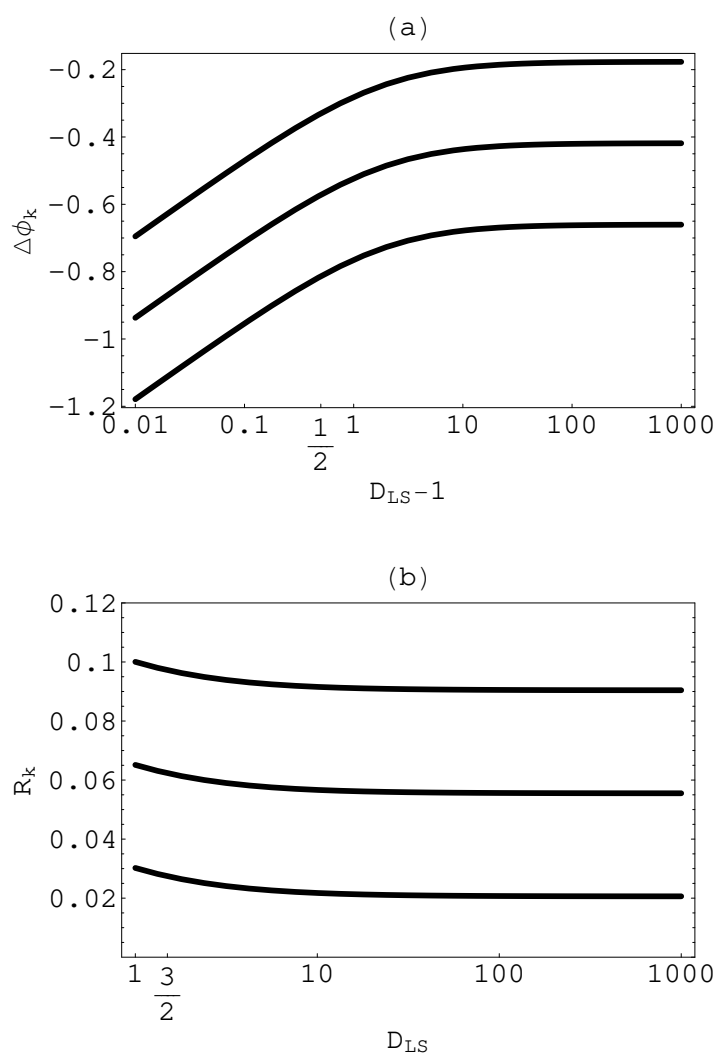

FIG. 6: (a) Drift of the center of the caustic from the optical axis as a function of the source distance; from the bottom up, the curves are for $k=2,3,4$, respectively. (b) Semiamplitudes of the caustics with $k=2,3,4$ (from bottom to top) as a function of the source distance. Both plots are done for $a=$ 0.1 and equatorial observer $\mu_{O}=0$.

(115) and (116). The result is shown in Fig. 7 where the caustic appears as a tube with the transverse section having the shape of a four cusped astroid. At large distances the caustic tube keeps its angular extension fixed and thus covers a larger and larger area. Close to the horizon, the caustic tube winds around the black hole indefinitely. A similar picture has already been done by Rauch \& Blandford [3] by numerical techniques for the caustic of order $k=1$. Our plot is entirely based on our analytical formulae (115) and (116), which are valid for arbitrary order except $k=1$. Our study is thus complementary to that of Ref. [3]. The origin of the logarithmic divergence in the caustic angular position can be traced back to the divergence of the integral $I_{2}$ in Eq. (76) (see also Eq. (B4) in Appendix B). Indeed, the integrand contains a factor $\Delta^{-1}$, which diverges linearly as the integration variable $r$ approaches the horizon. Since $D_{L S}$ is the lower bound of that integral, $I_{2}$ must diverge logarithmically as $D_{L S}$ tends to the horizon. The divergence of $I_{2}$ has no effect in Schwarzschild, since it appears multiplied by the black hole spin, but as soon as $a \neq 0$, the logarithmic divergence is transferred to the azimuthal

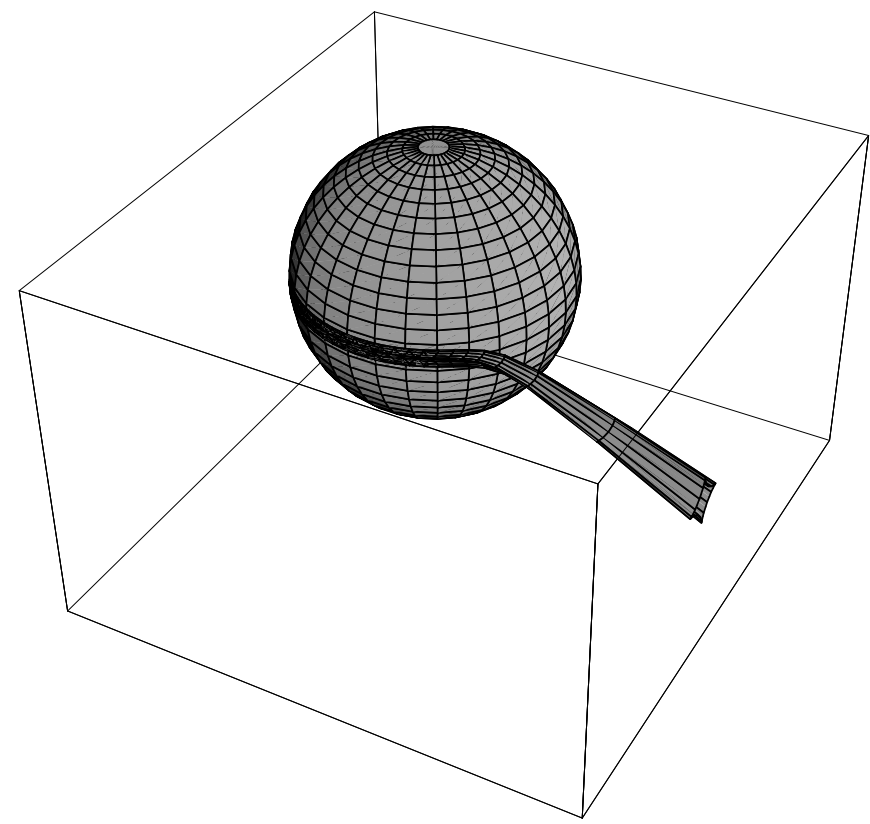

FIG. 7: A 3-dimensional view (in Boyer-Lindquist coordinates) of the whole caustic tube for $k=3, a=0.1$ and equatorial observer $\mu_{O}=0$. The sphere is the horizon of the black hole.

shift $\phi_{O}-\phi_{S}$, so that photons emitted by a source very close to the horizon must perform several loops before exiting. The latter argument is completely independent of our perturbative expansion in the black hole spin, proving that the logarithmic divergence is not an artifact of our perturbative framework. Moreover, the divergence of $I_{2}$ is not even a product of the strong deflection limit, since it is still there whatever the values of the constants of motion $J$ and $Q$. Therefore, it seems plausible to us that even the primary caustic tube (with $k=1$ ), which is not included in our treatment, should show a similar behavior, winding an infinite number of times before entering the horizon. This seems not to be observed in the numerical analysis by Rauch \& Blandford [3], where the primary caustic tube always appears to perform a finite number of loops before plunging into the horizon, except for the extremal case $a=0.5$. It is anyhow difficult to believe that the divergence is compensated by any of the remaining terms in Eq. (76) for intermediate values of $a$.

Our formulae (115) and (116) represent the transverse section of the caustic tube at fixed $D_{L S}$. One might be interested to a different transverse section, e.g. the section at fixed $\phi_{S}$. This is particularly interesting to study the approach of the caustic tube to the horizon, when the drift is large and $D_{L S}$ is very close to 1 . In this approximation, we can find the following expression for 
any fixed value of $\phi_{S}$

$$
\begin{aligned}
\mu_{S} & =(-1)^{k} \mu_{O} \pm R_{k}\left(D_{L S}=1\right) \sqrt{1-\mu_{O}^{2}}\left(1-\xi^{2}\right)^{3 / 2} \\
D_{L S} & =1+\frac{16}{9} e^{-\Delta \phi}\{1 \\
& \left.+\frac{a \sqrt{1-\mu_{O}^{2}}}{9}\left[2 c_{k}-\left(2 c_{k}+7 \sqrt{3}\right) \cos ^{3} \xi\right]\right\},(121)
\end{aligned}
$$

where $\Delta \phi=\phi_{S}-\Delta \phi_{k}\left(D_{L S}=\infty\right)$ is the difference between the chosen value of $\phi_{S}$ and the asymptotic drift of the caustic center. This expression is strictly valid for large values of $\Delta \phi$, but since the caustic drift sensibly increases only when $D_{L S}$ is very close to 1 , it is sufficient that $\Delta \phi>0.1$ in order to validate this expansion. Fig. 8 shows the transverse sections of a caustic tube obtained at different values of $\Delta \phi$. As the drift is increased, the caustic becomes thinner and thinner while it approaches the horizon.

\section{Gravitational lensing near caustics}

The higher order images of ordinary sources like stars or X-ray binaries are usually very faint, except for the event of a caustic crossing. Therefore, although in principle it is possible to analyze the lens equation in the general case, it is much more interesting to study the gravitational lensing of a source in the neighborhood of a caustic. This case is certainly the most relevant for observations and is worth a complete and detailed analysis.

\section{Position of the images}

The position of a source near a caustic can be expanded in the following way

$$
\begin{gathered}
\mu_{S}=(-1)^{k} \mu_{o}+\delta \mu_{S}, \\
\phi_{S}=(1-k) \pi-\Delta \phi_{k}+\delta \phi_{S},
\end{gathered}
$$

with $\delta \mu_{S}$ and $\delta \phi_{S}$ being of the same order of the caustic extension $R_{k}$, thus weighing as $a^{2}$ in the perturbative expansion.

Correspondingly, the solutions of the lens equation will be very close to the critical points. We can thus use the following expansion for $\psi$

$$
\psi=k \pi+\psi_{k}^{(1)}+\delta \psi
$$

with $\delta \psi$ being of order $a^{2}$.
Using these expansions in Eq. (105), $\delta \psi$ can be obtained as a function of the source position and $\xi$

$$
\begin{aligned}
\delta \psi & =-\left(1-\mu_{O}^{2}\right) \frac{\delta \phi_{S}}{\hat{\xi}}-\frac{16}{9} a^{2}\left(1-\hat{\xi}^{2}\right)-\frac{c_{k}}{2} a^{2}\left(3-2 \mu_{O}^{2}-\hat{\xi}^{2}\right) \\
& +\left[3 \sqrt{3} \sqrt{D_{L S}}\left(2 D_{L S}-3\right)\left(3+D_{L S}\right)^{3 / 2}\right]^{-1} \\
& \cdot\left\{\left(3-2 \mu_{O}^{2}-\hat{\xi}^{2}\right)\left(3+D_{L S}\right)\left(2 D_{L S}-3\right)\right. \\
& \cdot\left[9-2 D_{L S}-2\left(2 D_{L S}-3\right)\left(\sqrt{D_{L S}}-\sqrt{3+D_{L S}}\right)^{2}\right] \\
& \left.-4 D_{L S}\left(3+D_{L S}\right)\left(2 D_{L S}-3\right)\left(5-\hat{\xi}^{2}\right)+108 D_{L S} \hat{\xi}^{2}\right\},
\end{aligned}
$$

whereas $\xi$ is determined by the equation

$$
S(-1)^{k} \frac{\delta \mu_{S}}{\sqrt{1-\mu_{O}^{2}} \sqrt{1-\xi^{2}}}+\frac{\delta \phi_{S} \sqrt{1-\mu_{O}^{2}}}{\xi}+R_{k}=0
$$

in which $S= \pm 1$ is inherited by the sign ambiguity of the $(\psi, \xi)$ parametrization (see [22] for more details about the resolution of the sign ambiguity). Note that the lens equation formally remains the same as in the $D_{L S}=\infty$ case, though the position and the extension of the caustic have changed. The $\xi$ equation (126) can be easily put in the form of a fourth degree polynomial equation. It admits two solutions if the source is outside the caustic and four solutions if the source is inside the caustic. The solutions so obtained satisfy the original equation (126) with one definite choice of the $\operatorname{sign} S$, which is then univocally determined for each image.

Once we have the position of the image in the $(\psi, \xi)$ space, it is straightforward to write the position of the image in the observer sky. To this purpose, it is important to note that $\xi$ is known through Eq. (126) to zero order only. So, the position of the image on the observer sky is consistently determined to zero order as

$$
\begin{aligned}
& D_{O L} \theta_{1}=-\frac{3 \sqrt{3}}{2} \xi\left(1+\epsilon_{k}\right) \\
& D_{O L} \theta_{2}=S \frac{3 \sqrt{3}}{2} \sqrt{1-\xi^{2}}\left(1+\epsilon_{k}\right),
\end{aligned}
$$

with $\epsilon_{k}$ given by Eq. (71). The image appears on the critical ring of order $k$ at a position angle $\arcsin \xi$ and half-sky determined by the sign $S$.

\section{Brightness of a lensed image}

In typical gravitational lensing studies, the change in the apparent brightness of the source is simply given by the geometrical magnification, defined as the ratio of the elementary angular area of the image and the angular area of the source as it would be seen without any gravitational lensing. 


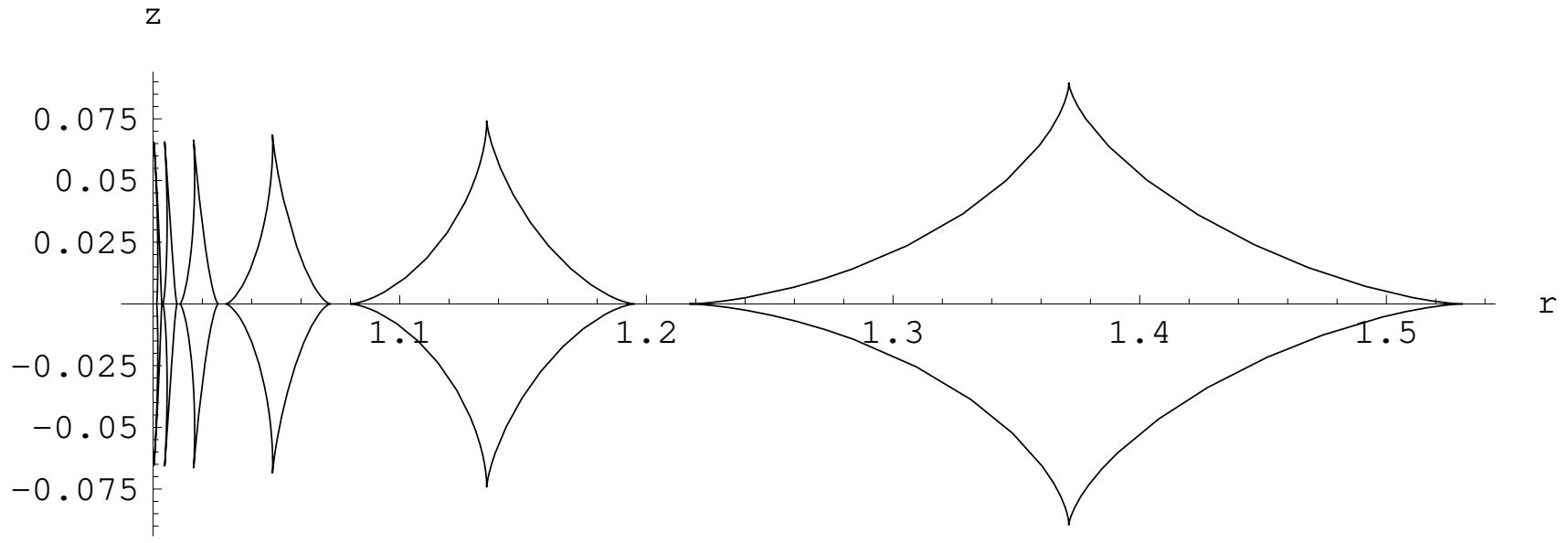

FIG. 8: Transverse sections of the caustic tube for $k=3, a=0.1$ and $\mu_{O}=0$ obtained at $\delta \phi=0.2,0.3,0.4,0.5,0.6,0.7$ going from the right to the left. The abscissa is the Boyer-Lindquist radial coordinate, whereas the ordinate is the pseudo-euclidean coordinate $z=r \cos \vartheta$.

When the source is far from the lens, this definition makes sense, since the background metric is asymptotically flat and we can speak of a source without the lens by simply re-interpreting the source coordinates as coordinates in the asymptotic Minkowski metric. This procedure loses any meaning when the source is deeply within the gravitational field of the black hole. Moreover, the frequency of the emitted photon does not coincide with the frequency detected by the observer because of gravitational redshift. Conservation of the photon number warrants that the quantity $I / \nu^{3}$ is conserved on a bundle of light rays, with $I$ being the specific intensity, defined as the energy $d E$ crossing a surface element $d A$ pointing an angular area $d \Omega$ in the time interval $d t$ and frequency interval $d \nu$.

In order to build a simulated lensed image of a source close to a black hole, one just needs to find the position of the image for any point belonging to the source and determine the specific intensity observed at that point. The position of the higher order images for any given source position can be read from Eqs. (127) and (128), whereas the specific intensity measured by the observer is related to the specific intensity emitted by the source through the relativistic relation

$$
I_{o}=\frac{\nu_{o}^{3}}{\nu_{e}^{3}} I_{e}
$$

where the redshift factor can be calculated as usual as

$$
\frac{\nu_{o}}{\nu_{e}}=\frac{p_{0}}{u^{\mu} p_{\mu}}
$$

with $p_{\mu}=g_{\mu \nu} \dot{x}^{\nu}$ being the momentum of the photon and $u^{\mu}$ being the 4 -velocity of the emitting particle. In stationary spherically symmetric and in Boyer-Lindquist coordinates for the Kerr metric, $\partial_{t}$ is a Killing vector and thus $p_{0}$ is a conserved quantity (we have put it to 1 by a choice of the affine parameter).
So, for any point of the source we can find the location of the corresponding high order images and if any model provides us the specific intensity of the source at that point, we can calculate the specific intensity as seen by the observer.

In the case in which the source is transparent, it can be conveniently characterized by its volume emissivity $j_{\nu}\left(x^{\mu}, \hat{p}_{\mu}\right)$, defined as the energy $d E$ in the frequency interval $d \nu_{e}$ emitted by the proper spacetime volume $\sqrt{-g} d^{4} x$ centered on $x^{\mu}$ in a solid angle $d \Omega$ centered on the direction given by the vector $\hat{p}_{\mu}$.

If we want the specific intensity measured by the observer in the sky direction $\left(\theta_{1}, \theta_{2}\right)$, we just have to trace back the null geodesic reaching the observer with such a direction and sum up the contributions given by all source elements along this geodesic. We thus have 35]

$$
I_{o}=\int \frac{\nu_{o}^{3}}{\nu_{e}^{3}} j_{\nu}\left(x^{\mu}, \dot{x}_{\mu}\right) d l_{\text {prop }},
$$

where $d l_{\text {prop }}$ is the geodesic line element as measured in the emitter frame.

\section{CONCLUSIONS}

Year after year, our knowledge on the environment surrounding the supermassive black holes is growing at a higher and higher rate, thanks to the surprising development of the technology related to high resolution observations. Angular resolutions of the order of the microarcsecond are now reachable in the radio band and sooner or later will be reached in the sub-mm and X-ray band. At the same time, the interferometric observations in the infrared at the Keck telescopes and at VLT are unveiling a very rich stellar enviroment around the Galactic center, which is drawing more and more interest. High resolution observations are the premise for the discovery 
of possible signatures of general relativity effects from supermassive black holes, which would open a new era in the understanding of gravitational physics.

Within this context, the comprehension of the propagation of photons in a strong field environment is of capital importance. Many numerical codes partially exploiting the analytical solutions of Kerr geodesics are available and have been used to build simulated images of the black holes. At the same time, analytical studies have been complementarily developed to conquer precious insight about the mathematical structure of the lens mapping in this very special gravitational lensing framework.

The strong deflection limit allows to study gravitational lensing in the extreme situation of photons travelling very close to the unstable circular orbit around the black hole. Such photons give rise to an infinite sequence of additional images which contribute to the total flux received by the observer by a non-negligible amount (see Ref. [6] for an estimate of their relevance). Therefore, the study of such images is far from being a mere academic exercise, but acquires a striking importance by the fact that these images carry invaluable information about the strong gravitational fields around the black hole. Whereas complete numerical studies of these images are difficult because of the extreme accuracy required to follow photons travelling around the unstable circular orbit, analytical studies benefit from the great simplification introduced by the strong deflection limit. Therefore, higher order images represent a unique window where we can confront simple analytical results from General Relativity with observations.

In this paper we have removed the traditional limitation of the strong deflection limit to sources very far from the black hole. We have thus analytically explored extreme gravitational lensing of sources close to a black hole for the first time. There is no limitation to the validity of our results, which are applicable even to sources just outside the horizon.

In the spherically symmetric case, we have shown that the same formulae for the deflection of the photon can be applied both to sources outside the photon sphere and to sources inside the photon sphere, whose images appear inside the so-called shadow border. We have specified our formulae to the Schwarzschild case in order to test the validity of the strong deflection limit throughout the range of source distances.

For the Kerr black hole, the only modification comes in the resolution of the radial integrals in the geodesics equations. This brings to a modification of the size and shape of the critical curves. We have obtained a complete analytical description of the caustic tube from infinite distances up to the horizon, showing that the caustic tube winds indefinitely around the black hole because of a logarithmic divergence in the azimuthal geodesic equation. We have also updated the gravitational lensing of sources near a caustic.

The formulae contained in this paper can be applied to physically motivated models of sources around a super- massive black hole, where they can be used to calculate the shape and the brightness of the higher order images. We leave this interesting task to future work, contenting ourselves with the complete analytical derivation of all relevant formulae here.

\section{Acknowledgments}

The authors acknowledge support for this work by MIUR through PRIN 2006 Prot. 2006023491_003 and by research fund of the Salerno University.

\section{APPENDIX A: DIFFERENT PERTURBATIVE PARAMETERS FOR THE STRONG DEFLECTION LIMIT}

Recently Iyer and Petters 31] have rewritten Darwin's formula for the deflection angle of a Schwarzschild black hole in the strong deflection limit in terms of a new parameter $b^{\prime}$, defined as

$$
b^{\prime} \equiv 1-\frac{u_{m}}{u} .
$$

Recalling the definition of Darwin's perturbative parameter $\epsilon$ (19), we get the simple relation

$$
b^{\prime}=\frac{\epsilon}{1+\epsilon} .
$$

Of course, if we stop at the lowest order in the expansion, the deflection angle can be indifferently expressed in the equivalent forms

$$
\begin{aligned}
\alpha & =-\log \epsilon+\log \left[216(2-\sqrt{3})^{2}\right]-\pi+O(\epsilon) \\
& =-\log b^{\prime}+\log \left[216(2-\sqrt{3})^{2}\right]-\pi+O\left(b^{\prime}\right) .
\end{aligned}
$$

Both formulae are correct to lowest order, the differences being stored in the higher order terms in the perturbative expansions. Iyer and Petters have found that the higher order discrepancy between these two formulae and the exact deflection angle is significantly smaller for the $b^{\prime}$ formula than for Darwin's one [31]. Of course, by specifying an invertible function $f_{\lambda}(\epsilon)$ such that $f_{\lambda}(0)=0$ and $f_{\lambda}^{\prime}(0) \neq 0$, one can always define $\lambda \equiv f_{\lambda}(\epsilon)$ as a new perturbative parameter. By a suitable choice of $f_{\lambda}$ one can make the higher order corrections vanish up to an arbitrary order $n$. However, this normally corresponds to a more and more complex form of $f_{\lambda}$, which spoils the advantages of the perturbative expansion. The choice of the perturbative parameter is arbitrary within the mentioned constraints on $f_{\lambda}$, but is normally driven by some physical quantities that can be easily expressed in terms of the perturbative parameter.

In the case of gravitational lensing in the strong deflection limit, this point can be made clearer once we construct the formulae for the position of the images in 
the two perturbative frameworks. If we use the $b^{\prime}$ formula in the lens equation, we trivially obtain $b_{n}^{\prime}=\epsilon_{n}$ for the $n$-th image, with $\epsilon_{n}$ always given by the expression (54). The difference between the two frameworks actually emerges from the expression of the angular position in the observer sky $\theta$ in terms of the new parameter, which reads

$$
\theta=\frac{u}{D_{\mathrm{OL}}}=\theta_{m}(1+\epsilon)=\frac{\theta_{m}}{1-b^{\prime}} .
$$

If we plug $b_{n}^{\prime}=\epsilon_{n}$ directly into Eq. A5 we have a new formula for the position of the images

$$
\theta_{n}=\frac{\theta_{m}}{1-\epsilon_{n}}
$$

which can be compared to the classical formula by Darwin

$$
\theta_{n}=\theta_{m}\left(1+\epsilon_{n}\right)
$$

In both formulae $\epsilon_{n}$ is given by Eq. (54) and is just a function of the source position, with no memory of the perturbative framework used.

If $\epsilon_{n}$ is small, the two formulae are very close each other, the difference being order $\epsilon_{n}^{2}$ and thus completely negligible. If an $\epsilon_{n}^{2}$ accuracy is necessary, both formulae must be complemented by their respective higher order terms. Then the difference will be in the third order and so on.

On the other hand, in intermediate situations, in which $\epsilon_{n}$ is not small, Eq. (A6) does significantly better than Eq. (A7) and can be used to extend the range of validity of the first order expansion of the Strong Deflection Limit, without resorting to the second order terms.

\section{APPENDIX B: ADDITIONAL TERMS IN THE RADIAL INTEGRALS}

As anticipated in Section source and observer distances entirely comes from the resolution of the radial integrals (98) and (99). These integrals can be performed using the general tools of Section [I] and then expanded to second order in the black hole spin $a$. Actually, the integral $I_{2}$ is already multiplied by $a$ in Eq. (76), so that it is sufficient to stop at the first order in its expansion. The results are

$$
\begin{aligned}
& I_{1}=-a_{1} \log \delta+b_{1}+c_{1}\left(D_{L S}\right)+c_{1}\left(D_{O L}\right) \\
& I_{2}=-a_{2} \log \delta+b_{2}+c_{2}\left(D_{L S}\right)+c_{2}\left(D_{O L}\right)
\end{aligned}
$$

with the coefficients $a_{1}, b_{1}, a_{2}$, and $b_{2}$ being unchanged with respect to the appendix of Ref. [22]. To the second order in $a$, the function $c_{1}$ reads

$$
\begin{aligned}
c_{1}(r) & =\frac{a_{1}}{2} \log \left[(2+\sqrt{3}) \frac{\sqrt{3 r}-\sqrt{3+r}}{\sqrt{3 r}+\sqrt{3+r}}\right] \\
& +\frac{8 a \hat{\xi} \sqrt{r}}{3 \sqrt{3} \sqrt{3+r}(2 r-3)} \\
& +a^{2}\left[27(2 r-3)^{2} \sqrt{r}(3+r)^{3 / 2}\right]^{-1} \\
& \cdot\left\{2 ( 2 r - 3 ) ( 3 + r ) \left[2(2 r-3)(\sqrt{r}-\sqrt{3+r})^{2}\left(1-\hat{\xi}^{2}\right)\right.\right. \\
& \left.\left.+14 r+10 r \hat{\xi}^{2}-9\left(1-\hat{\xi}^{2}\right)+8 r \mu_{o}^{2}\right]-216 r \hat{\xi}^{2}\right\} . \quad(\mathrm{B} 3)
\end{aligned}
$$

To the first order in $a$, the function $c_{2}$ is

$$
\begin{aligned}
c_{2}(r) & =\frac{a_{2}}{2} \log \left[(2+\sqrt{3}) \frac{\sqrt{3 r}-\sqrt{3+r}}{\sqrt{3 r}+\sqrt{3+r}}\right] \\
& +\log \left[\frac{2 \sqrt{r}+\sqrt{3+r}}{3(2 \sqrt{r}-\sqrt{3+r})}\right] \\
& +\frac{2 a}{3 \sqrt{3}} \hat{\xi}[\sqrt{r} \sqrt{3+r}(2 r-3)]^{-1} \\
& \cdot\left[2(2 r-3)(\sqrt{r}-\sqrt{3+r})^{2}+18 r-9\right] .
\end{aligned}
$$

It can be easily checked that both functions vanish as their arguments go to infinity. Moreover, if $r \rightarrow 3 / 2$, the divergence in $c_{i}$ is compensated by the vanishing of $\delta$. As $r<3 / 2$, it is also easy to see that we must have $\delta^{2}<0$, analogously to the spherically symmetric case. The second logarithm in $c_{2}$ diverges at $r=1$. The implications of this divergence are discussed in Section
[1] A. Broderick and R. Blandford, MNRAS 342, 1280 (2003); 349, 994 (2004).

[2] B. Carter, Phys. Rev. 174, 1559 (1968).

[3] K.P. Rauch and R.D. Blandford, ApJ, 421, 46 (1994).

[4] A. Cadez, C. Fanton, and M. Calvani, New Astron. 3, 647 (1998); A. Cadez, M. Calvani, and C. Fanton, Mem. Soc. Astron. Italiana, 74, 446 (2003).

[5] S.U. Viergutz, A\&A 272, 355 (1993).

[6] K. Beckwith and Ch. Done, MNRAS 352, 353 (2004); 359, 1217 (2005).
[7] J.P. Luminet, A\&A 75, 228 (1979).

[8] H. Falcke, F. Melia, and E. Agol, ApJ 528, L13 (1999); A. de Vries, Class. Quant. Grav. 17, 123 (2000); R. Takahashi, Astrophys. J. 611, 996 (2004); K. Beckwith and C. Done, MNRAS 359, 1217 (2005).

[9] A.E. Broderick and A. Loeb, MNRAS 363, 353 (2005); 367, 905 (2006).

[10] C. Darwin, Proc. of the Royal Soc. of London A249, 180 (1959).

[11] R.D. Atkinson, Astron. Jour. 70, 517 (1965). 
[12] H.C. Ohanian, Amer. Jour. Phys. 55, 428 (1987).

[13] V. Bozza, S. Capozziello, G. Iovane, and G. Scarpetta, Gen. Relativ. Gravit. 33, 1535 (2001).

[14] R.J. Nemiroff, Amer. Jour. Phys. 61, 619 (1993).

[15] K.S. Virbhadra and G.F.R. Ellis, Phys. Rev. D 62, 084003 (2000).

[16] W. Hasse and V. Perlick, Jour. of Math. Phys. 47, 042503 (2006).

[17] A.O. Petters, MNRAS 338, 457 (2003).

[18] F. Melia and H. Falcke, Annual Review of Astronomy and Astrophysics 39, 309 (2001).

[19] F. Eisenhauer, R. Genzel, T. Alexander et al., ApJ 628, 246 (2005).

[20] M.P. Muno et al., ApJ 622, L113 (2005); M.P. Muno et al., arXiv:0707.1907.

[21] V. Bozza, F. De Luca, G. Scarpetta, and M.Sereno, Phys. Rev. D 72, 083003 (2005).

[22] V. Bozza, F. De Luca, and G. Scarpetta, Phys. Rev. D 74, 063001 (2006).

[23] V. Bozza, Phys. Rev. D 66, 103001 (2002).

[24] E.F. Eiroa, G.E. Romero, and D.F. Torres, Phys. Rev. D 66, 024010 (2002); A. Bhadra, Phys. Rev. D 67, 103009 (2003); E. F. Eiroa, Phys. Rev. D 71, 083010 (2005); R. Whisker, Phys. Rev. D 71, 064004 (2005); A. S. Majumdar and N. Mukherjee, Int. J. Mod. Phys.
D 14, 1095 (2005); J. M. Tejeiro and E. A. Larranaga, gr-qc/0505054, K. K. Nandi, Y.-Z. Zhang, and A. V. Zakharov, Phys. Rev. D74, 024020 (2006); K. Sarkar and A. Bhadra, Class. Quant. Grav. 23, 6101 (2006); F. Rahaman, M. Kalam and S. Chakraborty, arXiv:0705.0740.

[25] E.F. Eiroa, Phys. Rev. D73, 043002 (2006).

[26] N. Mukherjee and A.S. Majumdar, astro-ph/0605224

[27] V. Bozza and L. Mancini, Gen. Rel. and Grav. 36, 435 (2004).

[28] V. Bozza and M. Sereno, Phys. Rev. D73, 103004 (2006).

[29] V. Bozza, Phys. Rev. D 67, 103006 (2003).

[30] G.N. Gyulchev and S.S. Yazadjiev, Phys. Rev. D75, 023006 (2007).

[31] S.V. Iyer and A.O. Petters, arXiv:gr-qc/0611086

[32] C.-M. Claudel, K.S. Virbhadra and G.F.R. Ellis, Jour. of Math. Phys. 42, 818 (2001).

[33] S. Chandrasekhar, Mathematical Theory of Black Holes, Clarendon Press, Oxford (1983).

[34] V. Bozza and L. Mancini, ApJ 611, 1045 (2004).

[35] M. Jaroszyński and A. Kurpiewski, A\& A 326, 419 (1997).

[36] R.H. Boyer and R.W. Lindquist, Jour. of Math. Phys. 8, 265 (1967). 